JHU-TIPAC-920023

ENSLAPP-L-405-92

$\mathrm{MPI}-\mathrm{Ph} / 92-85$

October 1992

hep-th/9212155

\title{
Harmonic space and quaternionic manifolds
}

\author{
A. Galperin ${ }^{(a) \dagger}$, E. Ivanov ${ }^{(b) \dagger}$ and O. Ogievetsky ${ }^{(c) \ddagger}$ \\ (a) Department of Physics and Astronomy \\ Johns Hopkins University, Baltimore, MD 21218 \\ (b) Laboratoire de Physique Theorique ENSLAPP, ENS Lyon \\ 46 Allee d'Italie, F-69364 Lyon Cedex 07, France \\ ${ }^{(c)}$ Max-Planck-Institut für Physik, Werner-Heisenberg-Institut \\ Föhringer Ring 6, 8000 Munich 40, Germany
}

\begin{abstract}
We find a principle of harmonic analyticity underlying the quaternionic (quaternionKähler) geometry and solve the differential constraints which define this geometry. To this end the original $4 n$-dimensional quaternionic manifold is extended to a biharmonic space. The latter includes additional harmonic coordinates associated with both the tangent local $S p(1)$ group and an extra rigid $S U(2)$ group rotating the complex structures. Then the constraints can be rewritten as integrability conditions for the existence of an analytic subspace in the bi-harmonic space and solved in terms of two unconstrained potentials on the analytic subspace. Geometrically, the potentials have the meaning of vielbeins associated with the harmonic coordinates. We also establish a one-to-one correspondence between the quaternionic spaces and off-shell $N=2$ supersymmetric sigma-models coupled to $N=2$ supergravity. The general $N=2$ sigma-model Lagrangian when written in the harmonic superspace is composed of the quaternionic potentials. Coordinates of the analytic subspace are identified with superfields describing $N=2$ matter hypermultiplets and a compensating hypermultiplet of $N=2$ supergravity. As an illustration we present the potentials for the symmetric quaternionic spaces.
\end{abstract}

\footnotetext{
$\dagger$ On leave from the Laboratory of Theoretical Physics, JINR, Dubna, Head Post Office, P.O. Box 79, 101000 Moscow, Russia

$\ddagger$ On leave from P.N. Lebedev Physical Institute, Theoretical Department, 117924 Moscow, Leninsky prospect 53, Russia
} 


\section{Introduction}

In the present paper we complete a study of gauge theories with self-duality type constraints within the framework of harmonic space approach. This study was initiated in papers [1, 2] where the self-dual Yang-Mills fields and hyper-Kähler (HK) metrics (generalizing to $4 n$ dimensions the self-dual Einstein equations) were considered. There the concept of harmonic analyticity, originally introduced in the framework of $N=2$ supersymmetry [3], was applied to purely bosonic self-dual Yang-Mills and Einstein theories in the four-dimensional space $R^{4}$ and their generalizations to $R^{4 n}$.

The harmonic superspace approach [3] is based on an extension of the original real $N=2$ superspace by harmonics $u_{i}^{ \pm}, \quad u^{+i} u_{i}^{-}=1, \quad i=1,2$ parametrizing the sphere $S^{2}=S U(2) / U(1)$ (where $S U(2)$ is the automorphism group of $N=2$ supersymmetry). The reasons for this are: $(i)$ the extended superspace has an analytic subspace of smaller Grassmann dimension (which essentially involves harmonics); (ii) the main constraints of all $N=2$ supersymmetric theories can be interpreted as integrability conditions for this harmonic analyticity; (iii) these constraints can be solved in terms of some arbitrary analytic potentials.

Similarly, in self-dual Yang-Mills and Einstein theories one $(i)$ extends the original real $R^{4 n}$ space (whose tangent group is $S U(2) \times S p(n)$ and holonomy group is contained in $S p(n)$ ) by the sphere $S^{2}=S U(2) / U(1)$ coordinatized by the harmonics $u_{i}^{ \pm}$; $(i i)$ interprets the original equations as integrability conditions for the existence of an analytic subspace which essentially involves harmonics and contains a half of the original $R^{4 n}$ coordinates; (iii) solves these equations in terms of some arbitrary analytic potentials [1, 2]. Thus, analyticity means a half of anticommuting coordinates in supersymmetry and a half of commuting coordinates in self-dual theories. We emphasize that the harmonic analytic potentials encode the full information about the original (constrained) theory and provide a tool of classifying and computing HK metrics and self-dual Yang-Mills fields.

An interesting application of HK manifolds in field theory is connected with their role as the target spaces of $N=2$ supersymmetric sigma models [4]. The unconstrained formulation of these manifolds given in [2] makes this connection transparent. It turns out that the general superfield Lagrangian of $N=2$ sigma models derived in [5, 6] using the harmonic superspace approach, is in one-to-one correspondence with the HK potentials. The basic $N=2$ matter superfields (analytic $Q^{+}$hypermultiplets) together with harmonics $u_{i}^{ \pm}$are recognized as coordinates of the abovementioned analytic subspace of the extended HK target manifold $R^{4 n} \times S^{2}$. Both the target harmonic space and the harmonic superspace (on which the hypermultiplets $Q^{+}$are defined) contain the same set of harmonics $u_{i}^{ \pm}$. This implies identification of the automorphism group $S U(2)$ of $N=2$ supersymmetry with the rigid $S U(2)$ group rotating three complex structure of the target HK manifold.

Thus, the harmonic space formulation of HK geometry on the one hand, and the harmonic superspace description of $N=2$ matter on the other, make the relation between this geometry and $N=2$ supersymmetry as clear as that between the Kähler geometry and $N=1$ supersymmetry [7]. Moreover, the fact that the $N=2$ matter superfield Lagrangian of ref. [5, 6] is composed of HK potentials, provides a direct proof [2] that this Lagrangian describes the most general matter self-couplings in the rigid $N=2$ 
supersymmetry.

When $N=2$ sigma model is put in a supergravity background, its bosonic target space is known to be quaternionic [8] rather than hyper-Kähler as in the flat case. The most general off-shell action of such sigma models was constructed in 99 in the framework of harmonic superspace. By analogy with the rigid case, the relevant superfield Lagrangian was conjectured to be composed of unconstrained potentials underlying the quaternionic geometry. As was observed in [9, 10], this object reveals a number of new features compared to its rigid $N=2$ supersymmetric prototype. For instance, the harmonics which appear explicitly in the Lagrangian and so are expected to be related to the target space geometry do not coincide with the harmonics present in the harmonic superspace. Instead they are expressed through them and the compensating analytic superfield $q_{i}^{+}$of $N=2$ supergravity.

In order to understand the structure and symmetries of the superfield $N=2$ sigma model action of ref. 9] and to prove its conjectured status as the most general action of matter in local $N=2$ supersymmetry, one clearly needs an unconstrained geometric formulation of the quaternionic geometry similar to that of the HK case [2]. Such a formulation is given in the present paper.

Quaternionic manifolds (see, e.g. [11, 12, 13, 14]) are $4 n$ dimensional Riemannian manifolds with the holonomy group contained in $S p(1) \times S p(n)$. Locally, the quaternionic manifolds (like the HK manifolds ) have three almost complex structures that obey the well-known Clifford algebra relation. However, now these structures are not covariantly constant, the $S p(1)$ part of the holonomy group rotates them. So, a distinction from the HK case is that the complex structures on quaternionic manifolds are in principle not defined globally and cannot be chosen constant. Actually, the HK manifolds can be regarded as a degenerate class of the quaternionic ones whose holonomy group does not contain the $S p(1)$ factor. Below we shall consider the general case with the $S p(1)$ factor being nontrivial. As was shown in [8], this is the geometry inherent to the bosonic manifolds of $N=2$ sigma models in the supergravity background.

We will show that the quaternionic geometry, like the HK one, is determined by a harmonic analyticity preservation principle which naturally leads to unconstrained analytic potentials as the fundamental objects of this geometry. An important feature of the quaternionic case is that an interpretation of the basic constraints as integrability conditions for the preservation of harmonic analyticity becomes possible only after extending the original $4 n$ dimensional manifold by two sets of harmonic coordinates. One of them consists of the ordinary harmonics of an extra rigid $S U(2)$ acting on the compex structures in the same way as in the HK case. The other is new; it parametrizes the local $S p(1)$ group acting in the tangent space of the manifold. In the limiting case of HK manifolds (i.e., when the $S p(1)$ curvature of quaternionic manifolds is vanishing) the new coordinates coincide with central-charge coordinates introduced in [2] to solve the HK constraints in an algorithmic way. Thus the geometric meaning of the central-charge coordinates is naturally revealed in the framework of the harmonic space formulation of the quaternionic geometry. New harmonic variables will be shown to be directly related to the analytic compensating hypermultiplet of $N=2$ supergravity which thus acquires a nice geometric interpretation.

The paper is planned as follows. We begin in Sect. 2 with giving the basic notions 
of the quaternionic geometry in real $4 n$ dimensional space (or $\tau$ world). We write the defining constraints of this geometry in a form similar to the self-dual Yang-Mills and HK geometry constraints as they have been given in [1, 2]. In Sect. 3 we introduce the concept of bi-harmonic space and give the necessary technical details. In Sect. 4 we interpret the quaternionic geometry constraints as integrability conditions for the existence of a kind of harmonic analyticity in a bi-harmonic extension of the original manifold. Sect. 5 describes the procedure of passing to a new representation of these constraints where the underlying analyticity becomes manifest (so called $\lambda$-world representation). In Sect. 6 and 7 we study the consequences of the quaternionic geometry constraints in the $\lambda$-world. In the process of this study in Sect. 7 we naturally come to unconstrained analytic quaternionic potentials $\mathcal{L}^{+4}$ and $\mathcal{L}_{\mu}^{+}$which emerge as harmonic vielbeins in some $\lambda$-world covariant derivatives. We prove that all geometric quantities relevant to the quaternionic geometry are expressible, like in the HK case [2], in terms of these two basic objects (in fact only one of them, $\mathcal{L}^{+4}$, is essential; the second one, as in the HK case, is locally a pure gauge). In Sect. 8 we treat the simplest example of "maximally flat" $4 n$ dimensional quaternionic manifold, the homogeneous space $S p(n+1) / S p(1) \times S p(n)$. The limiting procedure allowing to reproduce the harmonic space formulation of HK manifolds of ref. [2] by a contraction of that for quaternionic manifolds is described in Sect. 9. In Sect. 10 we analyze the most general harmonic superspace off-shell action of $N=2$ matter in the supergravity background [9] from the point of view of the harmonic space approach to quaternionic geometry. We show that all objects entering the action (including the supergravity compensators) as well as the relevant equations of motion have a clear interpretation in the framework of this approach. Thus the latter visualizes the one-to-one correspondence between local $N=2$ supersymmetry and quaternionic geometry [8]. Finally, in Sect. 11 we give the explicit form of the basic analytic potential $\mathcal{L}^{+4}$ for all symmetric quaternionic spaces classified by Wolf [11].

Before turning to the presentation, it is useful to point out that in this paper, similarly to [1, 2], we mainly use the standard language of differential geometry which is widely used in superfield gauge theories and operates with such notions as vielbeins, curvatures, torsions, tangent space, etc. All concepts and results given below are certainly transferrable to the language of complex geometry and twistor theory (the latter has been used to solve self-dual Einstein and Yang-Mills equations in the pioneering papers [16], [17] and to study quaternionic manifolds in [13]). One of our aims here is to demonstrate that the harmonic analyticity which has already proved its efficiency in a wide range of problems, both in supersymmetric and purely bosonic theories, is appropriate for the quaternionic geometry as well. In our presentation we closely follow the logic and motivation of papers [1, 2] so the reader may consult them for simpler examples.

\section{Quaternionic geometry in the real basis}

We start with a $4 n$ dimensional Riemann manifold $M$ with local coordinates $\left\{x^{\mu k}\right\}, \mu=$ $1,2, \ldots 2 n ; k=1,2$. One of the definitions of quaternionic geometry [8, 13, 11, 14] restricts the holonomy group to a subgroup of $S p(1) \times S p(n)$. Hence we can choose from the very beginning the tangent group to be $S p(1) \times S p(n)$. So, the tensor fields defined on 
the manifold $\left\{x^{\mu k}\right\}$ undergo gauge transformations both in their $S p(n)$ and $S p(1)$ indices

$$
\begin{aligned}
\delta \phi_{\alpha \beta \ldots i j \ldots}(x) & \equiv \phi_{\alpha \beta \ldots i j \ldots}^{\prime}(x+\delta x)-\phi_{\alpha \beta \ldots i j \ldots}(x) \\
& =\tau_{\alpha}^{\alpha^{\prime}}(x) \phi_{\alpha^{\prime} \beta \ldots i j \ldots}(x)+\tau_{\beta}^{\beta^{\prime}}(x) \phi_{\alpha \beta^{\prime} \ldots i j \ldots}(x)+\ldots \\
& +\tau_{i}^{i^{\prime}}(x) \phi_{\alpha \beta \ldots i^{\prime} j \ldots}(x)+\tau_{j}^{j^{\prime}}(x) \phi_{\alpha \beta \ldots i j^{\prime} \ldots}(x)+\ldots \\
\delta x^{\mu i} & =\tau^{\mu i}(x) .
\end{aligned}
$$

Correspondingly, the covariant derivative is given by

$$
\mathcal{D}_{\alpha i}=e_{\alpha i}^{\mu k} \partial_{\mu k}-\omega_{\alpha i(\sigma \rho)} \Gamma^{(\sigma \rho)}-\omega_{\alpha i(l k)} \Gamma^{(l k)} \equiv \nabla_{\alpha i}-\omega_{\alpha i(\sigma \rho)} \Gamma^{(\sigma \rho)}-\omega_{\alpha i(l k)} \Gamma^{(l k)} .
$$

Here $\omega_{\alpha i(\sigma \rho)}$ and $\omega_{\alpha i(l k)}$ are the $S p(n)$ and $S p(1)$ connections, $\Gamma^{(\sigma \rho)}$ and $\Gamma^{(l k)}$ are $S p(n)$ and $S p(1)$ generators

$$
\left[\Gamma^{(\sigma \rho)}, \Gamma^{(\delta \kappa)}\right]_{A}{ }^{C}=\frac{1}{2}\left(\Omega^{\sigma \delta} \Gamma^{(\rho \kappa)}+\Omega^{\sigma \kappa} \Gamma^{(\rho \delta)}+\Omega^{\rho \delta} \Gamma^{(\sigma \kappa)}+\Omega^{\rho \kappa} \Gamma^{(\sigma \delta)}\right)_{A}{ }^{C}
$$

(the similar relation holds for $\Gamma^{(l k)}$, with the $S p(n)$ invariant tensor $\Omega^{\rho \delta}$ replaced by $\epsilon^{l k}$ ). As a rule we deal with the fundamental spinor representations of $S p(n)$ and $S p(1)$

$$
\left(\mathcal{D}_{\alpha i}\right)_{\beta n}{ }^{\beta^{\prime} n^{\prime}}=\delta_{\beta}^{\beta^{\prime}} \delta_{n}^{n^{\prime}} \nabla_{\alpha i}+\delta_{n}^{n^{\prime}} \omega_{\alpha i \beta}{ }^{\beta^{\prime}}+\delta_{\beta}^{\beta^{\prime}} \omega_{\alpha i n}{ }^{n^{\prime}} .
$$

The commutator of two covariant derivatives produces the $S p(n)$ and $S p(1)$ components of the curvature tensor

$$
\left[\mathcal{D}_{\alpha i}, \mathcal{D}_{\beta j}\right]_{\rho n}{ }^{\rho^{\prime} n^{\prime}}=\delta_{n}^{n^{\prime}} R_{\alpha i \beta j \rho}{ }^{\rho^{\prime}}+\delta_{\rho}^{\rho^{\prime}} R_{\alpha i \beta j n}{ }^{n^{\prime}} \equiv R_{\alpha i, \beta j \rho n}^{\rho^{\prime} n^{\prime}}
$$

Now, the requirement that the holonomy group of this $4 n$ dimensional Riemannian manifold (i.e. the group generated by the Riemann tensor) belongs to $S p(n) \times S p(1)$ is equivalent to the following covariant constraints

$$
\begin{aligned}
& R_{\alpha i \beta j \rho} \rho^{\rho^{\prime}}=\epsilon_{i j} R_{\alpha \beta ; \rho}^{\rho^{\prime}} \\
& R_{\alpha i \beta j n} n^{n^{\prime}}=\Omega_{\alpha \beta} R_{i j n}{ }^{n^{\prime}} .
\end{aligned}
$$

The difference from the analogous constraint defining the HK manifolds [2] lies in eq. (2.7). Its r.h.s. is vanishing in the HK case, while in the quaternionic case it corresponds to the nonvanishing $S p(1)$ part of the holonomy group.

The Bianchi identities combined with (2.6) and (2.7) imply

$$
\begin{aligned}
R_{(\alpha \beta)\left(\rho \beta^{\prime}\right)} & =R_{\left(\alpha \beta \rho \beta^{\prime}\right)}+\left(\Omega_{\beta \rho} \Omega_{\alpha \beta^{\prime}}+\Omega_{\alpha \rho} \Omega_{\beta \beta^{\prime}}\right) R \\
R_{(i j)(k l)} & =\left(\epsilon_{i k} \epsilon_{j l}+\epsilon_{i l} \epsilon_{j k}\right) R, \quad R=\mathrm{constant}
\end{aligned}
$$

where

$$
R \equiv \frac{1}{6} R_{(i j)}^{(i j)}, \quad R_{\alpha i \beta j}{ }^{\alpha i \beta j}=8 n(n+2) R .
$$

The constant $R$ can be positive or negative, the constraints (2.6), (2.7) do not fix its sign. It is easy to see from eqns (2.5) - (2.9) that quaternionic manifolds are Einstein manifolds 
(with cosmological constant proportional to $R$ ). Hence homogeneous quaternionic manifolds are compact in the case $R>0$ and noncompact if $R<0$ [15]. The scalar $S p(1)$ curvature is by definition positive and is given by $\left|R_{i j}{ }^{i j}\right|=6|R|$.

Note that for $n=1$ (four-dimensional manifolds) the relations (2.6) and (2.7) are satisfied trivially and place no restrictions on the geometry (correspondingly, the Bianchi identities do not require $R_{(i j k l)}$ to vanish). In this case one is led to impose the condition

$$
R_{(i j k l)}=0
$$

"by hand". This gives rise to the generic form (2.9) for $R_{(i j)(k l)}$ and provides the definition of the quaternionic geometry for $n=1$.

Thus, irrespective of the value of $n$, the basic constraint defining the quaternionic geometry can be written as follows

$$
\left[\mathcal{D}_{\alpha i}, \mathcal{D}_{\beta j}\right]_{\rho n}{ }^{\rho^{\prime} n^{\prime}}=-2 \delta_{\rho}^{\rho^{\prime}} \Omega_{\alpha \beta} R \Gamma_{(i j) n}{ }^{n^{\prime}}+\delta_{n}^{n^{\prime}} \epsilon_{i j}\left[R_{(\alpha \beta \rho} \rho^{\left.\rho^{\prime}\right)}-R\left(\Omega_{\beta \rho} \delta_{\alpha}^{\rho^{\prime}}+\Omega_{\alpha \rho} \delta_{\beta}^{\rho^{\prime}}\right)\right]
$$

with

$$
\Gamma_{(i j) n}^{n^{\prime}}=\frac{1}{2}\left(\epsilon_{i n} \delta_{j}^{n^{\prime}}+\epsilon_{j n} \delta_{i}^{n^{\prime}}\right) .
$$

Symmetrizing (2.12) with respect to $i, j$ one obtains an equivalent equation

$$
\left[\mathcal{D}_{\alpha(i}, \mathcal{D}_{\beta j)}\right]_{\rho n}{ }^{\rho^{\prime} n^{\prime}}=-2 \delta_{\rho}^{\rho^{\prime}} \Omega_{\alpha \beta} R \Gamma_{(i j) n}{ }^{n^{\prime}} .
$$

Similar equations in the self-dual Yang-Mills and HK geometries contain the vanishing r.h.s. [1] 2]. In fact, the HK manifolds are related to the quaternionic ones via contraction $R \rightarrow 0$ which means the vanishing of the $S p(1)$ curvature $\sim|R|$ (see also Sect.9).

We would like to understand eq. (2.14) as an integrability condition. However, the standard trick [1, 2] with multiplying the l.h.s. by harmonics $u^{+i} u^{+j}$ does not immediately lead to this interpretation. The reason is the presence of the nonvanishing scalar $S p(1)$ curvature $\sim|R| \neq 0$.

Our further strategy is as follows. First, we shall introduce the coordinates of tangent space $S p(1)$ group, thus representing the $S p(1)$ part of the tangent space group by differential operators acting on these new variables. Then the $S p(1)$ curvature term in (2.12), (2.14) will be reinterpreted as a component of the torsion associated with the new $S p(1)$ coordinates. After this, we shall introduce the ordinary harmonics corresponding to some extra rigid automorphism $S U(2)$ group rotating the $S p(1)$ indices. Then it will be shown that eq. (2.14) admits the interpretation of the integrability condition for the existence of an appropriate (covariantly) analytic subspace in such a "bi-harmonic" extension of the original $4 n$ dimensional manifold. Finally, we shall solve the constraints and find the unconstrained analytic potentials of the quaternionic geometry.

\section{$3 \quad$ Harmonic extension of the sphere $S^{2} \sim S p(1) / U(1)$}

Here we give the basic technical details on harmonic coordinates on the sphere $S^{2} \sim$ $S p(1) / U(1)$ and extension of the latter by extra harmonics $u^{ \pm i}$. 
We parametrize $S p(1)$ by the harmonics $v_{k}^{a}$, i.e. by the $S p(1)$ matrix in the doublet representaion:

$$
v_{k}^{a} v_{a}^{l}=-\delta_{k}^{l}, \quad v_{k}^{a} v_{b}^{k}=-\delta_{b}^{a}, \quad \delta v_{k}^{a}=\tau_{k}^{l}(x) v_{l}^{a} .
$$

The indices $i, j, k, \ldots$ correspond to the local $S p(1)$ group (cf. (2.1)), while the indices $a, b, \ldots$ are inert under this group. The $S p(1)$ generators are given by

$$
\Gamma^{(i j)}=\frac{1}{2}\left(v_{a}{ }^{i} \epsilon^{j k} \frac{\partial}{\partial v_{a}^{k}}+v_{a}^{j} \epsilon^{i k} \frac{\partial}{\partial v_{a}^{k}}\right)
$$

while the operators of the covariant differentiation on the $S p(1)$ group manifold by

$$
Z^{(a b)}=\frac{1}{2}\left(v_{k}^{a} \epsilon^{b c} \frac{\partial}{\partial v_{k}^{c}}+v_{k}^{b} \epsilon^{a c} \frac{\partial}{\partial v_{k}^{c}}\right)=-v_{k}^{a} v_{l}^{b} \Gamma^{(k l)}
$$

Both sets of the differential operators (3.2) and (3.3) form $S p(1) \sim S U(2)$ algebras and commute with each other, e.g

$$
\begin{gathered}
{\left[Z^{(a b)}, Z^{(c d)}\right]=\epsilon^{b d} Z^{(a c)}+\epsilon^{b c} Z^{(a d)}+(a \leftrightarrow b)} \\
{\left[Z^{(a b)}, \Gamma^{(i j)}\right]=0}
\end{gathered}
$$

Next, let us assume that on the right index $a$ of $v_{k}^{a}$ some extra rigid $S U(2)$ is realised (so $v_{k}^{a}$ is a kind of "bridge" relating the local tangent $S p(1)$ and this rigid $S U(2)$ )

$$
\delta v_{k}^{a}=-\left(\tau_{r}\right)_{b}^{a} v_{k}^{b}, \quad\left(\tau_{r}\right)_{b}^{a}=\text { const }
$$

and consider a "harmonic extension" of the space $\left\{x, v_{k}^{a}\right\}$ by the harmonics $u^{ \pm a}$ defined on $S U(2) / U(1)$ :

$$
\left\{x, v_{k}^{a}\right\} \Rightarrow\left\{x, v_{k}^{a}, u^{ \pm a}\right\}, \quad u^{+a} u_{a}^{-}=1, \overline{u_{a}^{-}}=u^{+a}, \delta u^{ \pm a}=-\left(\tau_{r}\right)_{b}^{a} u^{ \pm b} .
$$

Further we shall proceed in a close parallel with singling out analytic subspaces in the harmonic spaces and superspaces [1, 2, 3, 22].

First of all we pass to another basis of the bi-harmonic space (3.6)

$$
\begin{aligned}
\left\{x, v_{k}^{a}, u^{ \pm a}\right\} \Rightarrow\left\{x, v_{k}^{ \pm}, u^{ \pm a}\right\}, \quad v_{k}^{ \pm}=v_{k}^{a} u^{ \pm a}, v^{+k} v_{k}^{-}=1 \\
Z^{++}=-\frac{1}{2} Z^{(a b)} u_{a}^{+} u_{b}^{+}=v_{k}^{+} \frac{\partial}{\partial v_{k}^{-}} \\
Z^{--}=\frac{1}{2} Z^{(a b)} u_{a}^{-} u_{b}^{-}=v_{k}^{-} \frac{\partial}{\partial v_{k}^{+}} \\
Z^{0}=Z^{(a b)} u_{a}^{+} u_{b}^{-}=v_{k}^{+} \frac{\partial}{\partial v_{k}^{+}}-v_{k}^{-} \frac{\partial}{\partial v_{k}^{-}} .
\end{aligned}
$$

The commutators between the $v$-derivatives $Z(3.8)$ and the non-vanishing commutators of the latter with the harmonic derivatives $\partial^{++}=u^{+a} \partial / \partial u^{-a}, \partial^{--}=u^{-a} \partial / \partial u^{+a}$ and $\partial^{0}=u^{+a} \partial / \partial u^{+a}-u^{-a} \partial / \partial u^{-a}$ are given by

$$
\left[Z^{++}, Z^{--}\right]=Z^{0}, \quad\left[Z^{0}, Z^{ \pm \pm}\right]= \pm 2 Z^{ \pm \pm}
$$




$$
\begin{gathered}
{\left[\partial^{++}, Z^{--}\right]=Z^{0}, \quad\left[\partial^{++}, Z^{0}\right]=-2 Z^{0}} \\
{\left[\partial^{--}, Z^{++}\right]=-Z^{0}, \quad\left[\partial^{--}, Z^{0}\right]=2 Z^{--}} \\
{\left[\partial^{0}, Z^{ \pm \pm}\right]= \pm 2 Z^{ \pm \pm} .}
\end{gathered}
$$

One more change of variables leads us to the following "analytic" basis in the space $\left\{x, v_{k}^{a}, u^{ \pm a}\right\}$ :

$$
\begin{aligned}
\left\{x, v_{k}^{a}, u^{ \pm a}\right\} \Rightarrow\left\{x, v_{k}^{ \pm}, u^{ \pm a}\right\} \Rightarrow\left\{x, z^{++}, z^{--}, z^{0}, w^{+a}, w^{-a}\right\} \\
z^{++}=\frac{v^{++}}{v^{-+}}, z^{--}=v^{-+} v^{--}, z^{0}=v^{-+}, v^{ \pm \pm}=u_{k}^{ \pm} v^{ \pm k} \\
v^{-+}=u_{k}^{-} v^{+k}, v^{+-}=u_{k}^{+} v^{-k}, v^{++} v^{--}-v^{-+} v^{+-}=1 \\
w_{a}^{+}=u_{a}^{+}-z^{++} u_{a}^{-}, w_{a}^{-}=u_{a}^{-}, v_{k}^{+}=-z^{0} w_{k}^{+}, v_{k}^{-}=-\frac{1}{z^{0}}\left(w_{k}^{-}+z^{--} w_{k}^{+}\right) .
\end{aligned}
$$

It is instructive to see how the original local $S p(1)$ group is realised in this basis

$$
\begin{aligned}
\delta z^{++} & =\tau^{i j}(x) w_{i}^{+} w_{j}^{+} \equiv \hat{\tau}^{++}(x, w), \delta z^{--}=\hat{\tau}^{--}+2 \hat{\tau}^{+-} z^{--}, \delta z^{0}=\hat{\tau}^{+-} z^{0} \\
\delta w_{a}^{+} & =-\hat{\tau}^{++} w_{a}^{-}, \quad \delta w_{a}^{-}=0 .
\end{aligned}
$$

Comparing with the results of ref. [9, 10], one observes that the newly introduced harmonics $w^{ \pm i}$ are composed of $u^{ \pm a}$ and behave under the local $S p(1)$ group precisely in the same manner as the harmonics $w^{ \pm a}$ entering the general $N=2$ matter Lagrangian in a $N=2$ supergravity background are composed of the $N=2$ harmonic superspace $u$ 's and behave under the transformations called in [10] quaternionic (analogs of the Kähler [7] and hyper-Kähler [10, 2] transformations). The object $z^{++}$stands for the supergravity compensator $N^{++}$. As we will see later on, this analogy is by no means accidental (see Sect. 10).

From (3.14) one concludes that $z^{++}, z^{--}, z^{0}$ transform independently of each other, i.e. each transforms through itself, $x$ and $w^{ \pm a}$. So, one can extract from (3.11) invariant subspaces containing smaller number of $z$ 's and overlapping over the harmonic part $\left\{w^{ \pm a}\right\}$ which in its own right constitutes an invariant subspace (together with $x$ ). In particular, there exist three invariant subspaces each including only one of the $z$ coordinates

$$
\left(x, z^{++}, z^{--}, z^{0}, w^{ \pm a}\right) \Rightarrow\left\{\left(x, z^{++}, w^{ \pm a}\right),\left(x, z^{--}, w^{ \pm a}\right),\left(x, z^{0}, w^{ \pm a}\right)\right\} \Rightarrow\left\{\left(x, w^{ \pm a}\right)\right\}(3
$$

This structure of the bi-harmonic space manifests itself in the form of the covariant derivatives in the analytic basis

$$
\begin{aligned}
& Z^{++}=\left(z^{0}\right)^{2} \frac{\partial}{\partial z^{--}} \\
& Z^{--}=\left(z^{0}\right)^{-2}\left\{z^{--}\left(z^{0} \frac{\partial}{\partial z^{0}}+z^{--} \frac{\partial}{\partial z^{--}}\right)-\frac{\partial}{\partial z^{++}}+\partial_{w}^{--}\right\}
\end{aligned}
$$




$$
\begin{aligned}
Z^{0} & =z^{0} \frac{\partial}{\partial z^{0}} \\
\mathcal{D}^{++} & =\partial_{w}^{++}-z^{++}\left[\mathcal{D}^{0}-z^{0} \frac{\partial}{\partial z^{0}}-z^{++} \frac{\partial}{\partial z^{++}}\right]+\left[\left(z^{0}\right)^{2}-1\right] \frac{\partial}{\partial z^{--}} \\
\mathcal{D}^{--} & =Z^{--}+\frac{\partial}{\partial z^{++}} \\
\mathcal{D}^{0} & =\partial_{w}^{0}+2\left(z^{++} \frac{\partial}{\partial z^{++}}-z^{--} \frac{\partial}{\partial z^{--}}\right) .
\end{aligned}
$$

Considering a general field on the bi-harmonic space, $\phi(x, z, w)$ (we discard possible external indices), we may impose on it the following $S p(1)$ covariant analyticity constraints

$$
\begin{aligned}
Z^{++} \phi=0 & \Rightarrow \frac{\partial}{\partial z^{--}} \phi=0, \\
\text { or } Z^{0} \phi=0 & \Rightarrow \frac{\partial}{\partial z^{0}} \phi=0, \\
\text { or } \quad\left(Z^{--}-\mathcal{D}^{--}\right) \phi=0 & \Rightarrow \frac{\partial}{\partial z^{++}} \phi=0,
\end{aligned}
$$

or any combination of them simultaneously. Indeed, any pair of the corresponding differential operators is closed under commutation; this follows also from (3.9), (3.10). The covariance of eqs. (3.18) with respect to $S p(1)$ transformations follows from the transformation properties of the derivatives involved

$$
\delta \frac{\partial}{\partial z^{0}}=0, \delta \frac{\partial}{\partial z^{++}}=0, \delta \frac{\partial}{\partial z^{--}}=-2 \hat{\tau}^{+-} \frac{\partial}{\partial z^{--}} .
$$

The solutions of these constraints are the fields living on the aforementioned invariant subspaces of the bi-harmonic space. In particular, three subspaces indicated in eq. (3.15) correspond to imposing first and second, second and third, first and third of the constraints (3.18), respectively. The fields on the subspace $\left(x, w^{ \pm a}\right)$ are singled out by employing the whole set of these constraints.

Now we will show that an arbitrary field $f_{i j k \ldots . .}(x)$ (having the standard transformation properties (2.1) under local $S p(1)$ ) can be rewritten as a constrained analytic field on the above bi-harmonic space. Correspondingly, the group $S p(1)$ is realised on such fields by the differential operators $Z^{(i j)}$.

Converting indices $i, j, k, \ldots$ of the field $f_{i j k \ldots}(x)$ with the harmonics $v_{i}^{a}$ we form a field which is inert under the local $S p(1)$ and transforms only under the rigid $S U(2)$ acting on the indices $a, b, c, \ldots$

$$
f_{a b \ldots c}(x, v)=v_{a}^{i} v_{b}^{j} \ldots v_{c}^{k} f_{i j \ldots k}(x) .
$$

Now the $S p(1)$ transformation of $f_{i j \ldots k}(x)$ is induced by the transformation of the variables $v_{a}^{i}$ and so it is represented by the differential operator $\Gamma^{(k l)}(3.2)$

$$
\delta^{*} f(x, v) \equiv f^{\prime}(x, v)-f(x, v)=\tau_{k}^{l}(x) \Gamma_{l)}^{(k} f(x, v) .
$$

Further, projecting $f_{a b \ldots c}(x, v)$ onto the monomials $\left(u^{+a} \ldots u^{+c}\right)$ one defines

$$
\begin{aligned}
f^{+n}\left(x, w, z^{0}\right) & =(-1)^{n} v^{+i_{1}} \ldots v^{+i_{n}} f_{i_{1} \ldots i_{n}}(x) \\
& =\left(z^{0}\right)^{n} w^{+i_{1}} \ldots w^{+i_{n}} f_{i_{1} \ldots i_{n}}(x) \\
& \equiv\left(z^{0}\right)^{n} \tilde{f}^{+n}(x, w) .
\end{aligned}
$$


These functions satisfy the relations

$$
\begin{aligned}
\mathcal{D}^{++} f^{+n} & =\partial_{w}^{++} f^{+n}=0 \\
\mathcal{D}^{0} f^{+n} & =n f^{+n} \\
Z^{0} f^{+n} & =n f^{+n} \\
Z^{++} f^{+n} & =0 \\
\left(Z^{--}-\mathcal{D}^{--}\right) f^{+n} & =\frac{\partial}{\partial z^{++}} f^{+n}=0
\end{aligned}
$$

and transform in the following way under the local $S p(1)$

$$
\begin{aligned}
\delta f^{+n}\left(x, w, z^{0}\right) & =f^{+n^{\prime}}\left(x^{\prime}, w^{\prime}, z^{0^{\prime}}\right)-f^{+n}\left(x, w, z^{0}\right)=0 \\
\delta \tilde{f}^{+n}(x, w) & =\tilde{f}^{+n^{\prime}}\left(x^{\prime}, w^{\prime}\right)-\tilde{f}^{+n}(x, w)=-n \hat{\tau}^{+-} \tilde{f}^{+n}(x, w) .
\end{aligned}
$$

To identify the place which $f^{+n}$ occupy among general functions on the bi-harmonic space, we give here a general characterisation of such functions.

By analogy with the harmonic functions on the ordinary sphere $S^{2}$ we restrict ourselves to the following functions $f^{(p, q)}(z, w)$ (for brevity, we omit the $x$ dependence)

$$
\begin{aligned}
& \mathcal{D}^{0} f^{(p, q)}(z, w)=q f^{(p, q)}(z, w) \\
& Z^{0} f^{(p, q)}(z, w)=p f^{(p, q)}(z, w)
\end{aligned}
$$

which corresponds to considering invariant functions on $S^{2} \times S^{2} \equiv S p(1) / U(1) \times S U(2) / U(1)$. In the basis $(z, w)$ the condition (3.24) is easy to solve: it simply states that $f^{(p, q)}(z, w)$ is a homogeneous function of degree $p$ with respect to $z^{0}$

$$
f^{(p, q)}(z, w)=\left(z^{0}\right)^{p} \tilde{f}^{(p, q)}\left(z^{++}, z^{--}, w\right), \quad \delta \tilde{f}^{(p, q)}=-p \hat{\tau}^{+-} \tilde{f}^{(p, q)} .
$$

Using the analyticity conditions (3.18), we may now covariantly constrain, in one way or another, the dependence of $f^{(p, q)}(z, w)$ on $z^{++}, z^{--}$. The simplest possibility is to completely eliminate this dependence

$$
Z^{++} f_{(1)}^{(p, q)}=\left(Z^{--}-\mathcal{D}^{--}\right) f_{(1)}^{(p, q)}=0 \Rightarrow \tilde{f}_{(1)}^{(p, q)}=\tilde{f}_{(1)}^{(p, q)}(w)
$$

However, as it follows from (3.9), ordinary harmonic derivatives $\mathcal{D}^{++}, \mathcal{D}^{--}$commute not with all operators defining the above analyticity. So, the result of action of these derivatives on $f_{(1)}^{(p, q)}$ is not in general again the field of the type (3.26), e.g.

$$
\begin{aligned}
& \mathcal{D}^{++} f_{(1)}^{(p, q)}=\left[\partial_{w}^{++}+z^{++}\left(Z^{0}-\mathcal{D}^{0}\right)\right] f_{(1)}^{(p, q)}(w, z) \\
& \mathcal{D}^{--} f_{(1)}^{(p, q)}=\left(z^{0}\right)^{-2}\left[\partial_{w}^{--}+z^{--} Z^{0}\right] f_{(1)}^{(p, q)}
\end{aligned}
$$

These fields are of the type (3.26) only provided $q=p=0$. One may still achieve this property for $\mathcal{D}^{++} f_{(1)}^{(p, q)}$ under a weaker condition

$$
\left(Z^{0}-\mathcal{D}^{0}\right) f_{(1)}^{(p, q)}=0 \Rightarrow q=p
$$


In this case the field

$$
\mathcal{D}^{++} f_{(1)}^{(q)}\left(w, z^{0}\right)=\partial_{w}^{++} f_{(1)}^{(q)}\left(w, z^{0}\right)
$$

is covariant under $S p(1)$ transfromations, which can be checked explicitly, using

$$
\delta \partial_{w}^{++}=-\hat{\tau}^{++}\left(Z^{0}-\mathcal{D}^{0}\right)-2 \hat{\tau}^{+-} \frac{\partial}{\partial z^{--}} .
$$

We call the fields satisfying (3.26), (3.27) the fields of the first kind. The functions $f^{+n}\left(w, z^{0}\right)$ considered before belong just to this class, they are singled out by the additional covariant constraint

$$
\mathcal{D}^{++} f_{(1)}^{(q)}\left(w, z^{0}\right)=\partial_{w}^{++} f_{(1)}^{(q)}\left(w, z^{0}\right)=0 \Rightarrow f_{(1)}^{(q)}\left(w, z^{0}\right)=\left(z^{0}\right)^{q} w^{+i_{1}} \ldots w^{+i_{q}} f_{i_{1} \ldots i_{q}} .
$$

Another interesting class of functions having a restricted $z^{++}, z^{--}$dependence is defined by the constraints

$$
\begin{aligned}
\left(Z^{0}-\mathcal{D}^{0}\right) f_{(2)}^{(p, q)} & =0 \Rightarrow f_{(2)}^{(p, q)} \equiv f_{(2)}^{(q)} \\
\left(\mathcal{D}^{--}-Z^{--}\right) f_{(2)}^{(p, q)}=\frac{\partial}{\partial z^{++}} f_{(2)}^{(q)} & =0 \\
\left(Z^{++}-\mathcal{D}^{++}\right) f_{(2)}^{(q)}=\left(\frac{\partial}{\partial z^{--}}-\partial_{w}^{++}\right) f_{(2)}^{(q)} & =0 .
\end{aligned}
$$

These fields do not depend on $z^{++}$, while their $z^{--}$dependence is restricted by

$$
\frac{\partial}{\partial z^{--}} f_{(2)}^{(q)}=\partial_{w}^{++} f_{(2)}^{(q)}
$$

so any such field is actually a collection of all the degrees of the harmonic derivative $\partial_{w}^{++}$ of the lowest component in its $z^{--}$expansion. So, we may limit our consideration to the component $\tilde{f}_{(2)}^{(q)} \|$ where the vertical line means the value at $z^{--}=0$. It transform as follows

$$
\delta \tilde{f}_{(2)}^{(q)}\left\|=-q \hat{\tau}^{+-} \tilde{f}_{(2)}^{(q)}\right\|-\hat{\tau}^{--} \partial_{w}^{++} \tilde{f}_{(2)}^{(q)} \| .
$$

The important difference of the second kind fields from the first kind ones consists in that $\partial_{w}^{++} \tilde{f}_{(2)}^{(q)}$ is again the field of the second kind, its $S p(1)$ weight and $U(1)$ charge being equal to $q+2$,

$$
\delta\left(\partial_{w}^{++} \tilde{f}_{(2)}^{(q)}\right)\left\|=-(q+2) \hat{\tau}^{+-}\left(\partial_{w}^{++} \tilde{f}_{(2)}^{(q)}\right)\right\|-\hat{\tau}^{--} \partial_{w}^{++}\left(\partial_{w}^{++} \tilde{f}_{(2)}^{(q)}\right) \|
$$

while $\partial_{w}^{++} \tilde{f}_{(1)}^{(q)}$ is a field of a new type with the nonaltered $S p(1)$ weight $q$ and the $U(1)$ charge shifted by 2 :

$$
\delta\left(\partial_{w}^{++} \tilde{f}_{(1)}^{(q)}\right)=-q \hat{\tau}^{+-}\left(\partial_{w}^{++} \tilde{f}_{(1)}^{(q)}\right) .
$$

(it belongs to the type (3.26)). 
Concluding this Section, we comment on several points which are important for understanding what we will do in the sequel.

As a first remark we point out that the bi-harmonic space $\{x, v, u\}$ contains evident subspaces $\left\{x, v^{+}, v^{-}\right\}$and $\left\{x, u^{+}, u^{-}\right\}$which are trivially closed both under the local $S p(1)$ and rigid $S U(2)$ transformations. As we will see later, these subspaces bear no direct relevance to the quaternionic geometry constraints, in contradistinction to the subspace $\left\{x, w^{+}, w^{-}\right\}$. Just the latter is preserved by these constraints, thus expressing most clearly the concept of harmonic analyticity in application to quaternionic geometry.

We also note that the functions given on the bi-harmonic space are in general represented by series which are not a naive double harmonic expansion with respect to the sets of harmonics $v^{ \pm i}$ and $u^{ \pm a}$. E.g., let us consider a function $f^{(q)}\left(x, w^{+}, w^{-}\right)$. It has a standard $w^{ \pm i}$-expansion

$$
f^{(q)}\left(x, w^{+}, w^{-}\right)=\sum f^{\left(i_{1} \ldots i_{n} j_{n+1} \ldots j_{n-q)}\right.}(x) w_{i_{1}}^{+} \ldots w_{i_{n}}^{+} w_{j_{n+1}}^{-} \ldots w_{j_{n-q}}^{-} .
$$

However, in the basis $\left(v^{ \pm i}, u^{ \pm a}\right)$ it is

$$
f^{(q)}(x, w)=\tilde{f}^{(q)}(x, v, u)=\sum f^{\left(i_{1} \ldots i_{n} j_{n+1} \ldots j_{n-q}\right)}(x) v_{i_{1}}^{+} \ldots v_{i_{n}}^{+} u_{j_{n+1}}^{-} \ldots u_{j_{n-q}}^{-}\left(-v^{-+}\right)^{-n} .
$$

The presence of unusual factors $\left(v^{-+}\right)^{-n}$ is necessary for ensuring the property

$$
Z^{0} f^{(q)}=\left(v^{+} \frac{\partial}{\partial v^{+}}-v^{-} \frac{\partial}{\partial v^{-}}\right) f^{(q)}=0
$$

in the basis $(v, u)$ (in the basis $(z, w)$ this property is evident). Clearly, (3.33) can never be achieved within a naive double $(v, u)$ harmonic expansion.

Having at our disposal the object $v^{-+}$with the vanishing $U(1)$ charge but with the unit $S p(1)$ weight, we are to take caution while transferring into the bi-harmonic space some statements valid in the ordinary harmonic space. In particular, the constraints

$$
\begin{aligned}
Z^{++} f^{(p, q)} & =v^{+} \frac{\partial}{\partial v^{-}} f^{(p, q)}=0 \\
Z^{0} f^{(p, q)} & =\left(v^{+} \frac{\partial}{\partial v^{+}}-v^{-} \frac{\partial}{\partial v^{-}}\right) f^{(p, q)}=p f^{(p, q)}
\end{aligned}
$$

do not imply that the $v^{-+}$expansion of $f^{(p, q)}$ terminates. In parallel with the standard term

$$
v^{+i_{1}} \ldots v^{+i_{p}} f^{(q-p)}(x, u)
$$

one is led to include into the $v$-decomposition of $f^{(p, q)}$ an infinite collection of the terms of the form

$$
v^{+i_{1}} \ldots v^{+i_{p}} v^{+i_{p+1}} \ldots v^{+i_{p+n}} f^{(q-p-n)}(x, u)\left(v^{-+}\right)^{-n}
$$

with $n \geq 1$. Each term of that sort is annihilated by $Z^{++}$and carries the $S p(1)$ weight $p$.

In what follows, to avoid subtleties of the bi-harmopnic decompositions, we will basically use the parametrization of the harmonic part of the bi-harmonic space by the 
coordinates $w^{ \pm i}, z^{++}, z^{--}, z^{0}$. We will assume standard harmonic expansions with respect to $w^{ \pm i}$, not specifying the dependence on $z^{++}, z^{--}$, except for a few special cases, including the important case when there is no such a dependence at all (as was already mentioned, the standard fields (2.1) belong just to this latter class). Recall that the $z^{0}$ dependence of the bi-harmonic fields is fixed as in eq. (3.21), as soon as we limit our consideration to the fields with a definite $S p(1)$ weight.

\section{Quaternionic geometry constraints as the integra- bility conditions for bi-harmonic analyticity}

Here we show that the defining constraint (2.14), being rewritten in the bi-harmonic extension of the original $4 n$ dimensional manifold $M$, can be given a natural interpretation of the integrability conditions for the existence of special analytic functions on this extended manifold.

Thus we deal with the bi-harmonic space

$$
\left\{x^{\mu i}, v_{a}^{l}, u_{a}^{ \pm}\right\}
$$

and treat ordinary fields (2.1) defined originally as unconstrained functions on $\left\{x^{\mu i}\right\}$ as fields (3.21) on the enlarged space (4.1). They may carry any number of external $S p(1)$ indices and an arbitrary $S p(1)$ weight (equal to their $U(1)$ charge). Their content is the same as that of (2.1) provided they satisfy the constraints (3.22). Of course, one is at liberty to consider more general functions on (4.1), in accordance with the analysis in the previous Section.

The main advantage of such an approach consists in the possibility to realize the generators of the tangent space local $S p(1)$ by differential operators. An equivalent representation of the covariant derivative (2.2) in the space (4.1) is given by

$$
\mathcal{D}_{\alpha a}=v_{a}{ }^{i} e_{\alpha i}^{\mu k} \partial_{\mu k}-v_{a}^{i} \omega_{\alpha i(\sigma \rho)} \Gamma^{(\sigma \rho)}-\frac{1}{2} v_{a}^{i} \omega_{\alpha i(l k)}\left(v_{b}^{l} \epsilon^{k t} \frac{\partial}{\partial v_{b}{ }^{t}}+v_{b}^{k} \epsilon^{l t} \frac{\partial}{\partial v_{b} t}\right) .
$$

One easily checks that the action of (4.2) on the field of the type (3.20) yields the correct covariant derivative of the coefficients $f_{i j \ldots k}(x)$

$$
\mathcal{D}_{\alpha a}\left(v_{a_{1}}^{i_{1}} v_{a_{2}}^{i_{2}} \ldots v_{a_{n}}^{i_{n}} f_{i_{1} i_{2} \ldots i_{n}}(x)\right)=v_{a}{ }^{i} v_{a_{1}}^{{ }^{i_{1}}} v_{a_{2}}{ }^{i_{2}} \ldots v_{a_{n}}^{{ }^{i_{n}}} \mathcal{D}_{\alpha i} f_{i_{1} i_{2} \ldots i_{n}}(x)
$$

It is easy to compute the commutator of two derivatives (4.2)

$$
\left[\mathcal{D}_{\alpha a}, \mathcal{D}_{\beta b}\right]_{\rho}^{\rho^{\prime}}=2 \delta_{\rho}^{\rho^{\prime}} \Omega_{\alpha \beta} R Z_{(a b)}+\epsilon_{a b}\{\}_{(\alpha \beta) \rho}^{\rho^{\prime}}
$$

where the braces stand for the $S p(n)$ curvature terms and $Z_{(a b)}$ is the covariant derivative with respect to the coordinates $v_{a}^{i}$ (it is defined in eqs. (3.3)). Now, the basic constraint (2.14) is written as

$$
\left[\mathcal{D}_{\alpha(a}, \mathcal{D}_{\beta b)}\right]_{\rho}^{\rho^{\prime}}=2 \delta_{\rho}{ }^{\rho^{\prime}} \Omega_{\alpha \beta} R Z_{(a b)}
$$


Digression. This is a good place to comment on the twistor space. Harmonics $v_{a}^{l}$ generate the sphere $S^{3}$ at each point of the quaternionic manifold $M$. Factorizing by $U(1)$ group acting on harmonics $v_{a}^{l}$ as $v_{1}^{l} \mapsto \exp (i \phi) v_{1}^{l}, v_{2}^{l} \mapsto \exp (-i \phi) v_{2}^{l}$ we obtain the space $S^{2}$ parametrized by complex structures at the point of $M$ : the complex structure corresponding to $v_{a}^{l}$ is

$$
I_{\alpha i}^{\beta j}=\left(v_{1 i} v_{2}^{j}+v_{2 i} v_{1}^{j}\right) \delta_{\alpha}^{\beta} .
$$

The space of all these complex structures attached at each point of $M$ is called the twistor space $\mathbf{Z}$ in 13. We conclude that the space $\left\{x^{\mu i}, v_{a}^{l}\right\}$ with the above equivalence relation is the twistor space of $M$.

The antiholomorphic direction with respect to the natural complex structure on $S^{2}$ is spanned by $Z_{11}$. We define now vector fields

$$
X_{\alpha}=v_{1}{ }^{i} e_{\alpha i}^{\mu k} \partial_{\mu k}-\frac{1}{2} v_{1}{ }^{i} \omega_{\alpha i(l k)}\left(v_{b}{ }^{l} \epsilon^{k t} \frac{\partial}{\partial v_{b}{ }^{t}}+v_{b}{ }^{k} \epsilon^{l t} \frac{\partial}{\partial v_{b}{ }^{t}}\right) .
$$

Using (4.5) one gets

$$
\left[X_{\alpha}, X_{\beta}\right]=2 \Omega_{\alpha \beta} R Z_{11}+v_{1}^{i}\left(\omega_{\beta i \alpha}^{\gamma}-\omega_{\alpha i \beta}^{\gamma}\right) X^{\gamma} .
$$

We have also $\left[Z_{11}, X_{\alpha}\right]=0$. Define now a tensor of an almost complex structure on the twistor space to have the space spanned by $\left\{X_{\alpha}, Z_{11}\right\}$ as its eigenspace with the eigenvalue $i$. The above arguments imply its integrability. This is the integrable complex structure discussed in [13].

In [13] for $R \neq 0$ a complex contact structure on $\mathbf{Z}$ was introduced. It can be also easily read off the equation (4.8). Let $\sigma$ be a local one-form satisfying $\left(\sigma, Z_{11}\right)=1$ and $\left(\sigma, X_{\alpha}\right)=0$ for all $\alpha$, where $(\sigma, X)$ is the value of the one-form $\sigma$ on a vector field $X$. The form $\sigma$ defines a complex contact structure on $Z$. To show this, one can write the explicit expression for $\sigma$ in the local coordinates. However, it is enough to use the identity $\left(d \sigma, X_{\alpha} \wedge X_{\beta}\right)=-\left(\sigma,\left[X_{\alpha}, X_{\beta}\right]\right)$. We find using (4.8) that

$$
\left(d \sigma, X_{\alpha} \wedge X_{\beta}\right)=-2 \Omega_{\alpha \beta} R .
$$

The kernel of $\sigma$ is spanned by $X_{\alpha}$. For $R \neq 0$ equation (4.9) shows that $d \sigma$ is nondegenerate on the kernel of $\sigma$. Therefore, $\sigma$ is a well-defined complex contact one-form.

Now we return to the main line of our exposition. What we have gained at this step is that the local $S p(1)$ indices $i, j, \ldots$ have been replaced by the rigid $S U(2)$ ones $a, b, \ldots$ and the quaternionic geometry constraint has been reformulated entirely in terms of appropriate covariant derivatives. Now we may proceed in the way well known from the previous examples of utilyzing the harmonic analyticity [1, 2, 3, 22]: multiply (4.5) by the extra harmonics $u^{+a}, u^{+b}$ and recast this relation into the form of the integrability condition

$$
\left[\mathcal{D}_{\alpha}^{+}, \mathcal{D}_{\beta}^{+}\right]=-2 \delta_{\rho}^{\rho^{\prime}} \Omega_{\alpha \beta} R Z^{++}, \quad \mathcal{D}_{\alpha}^{+}=u^{+a} \mathcal{D}_{\alpha a}, \quad Z^{++}=-u^{+a} u^{+b} Z_{(a b)} .
$$

The full set of commutators in this new representation can be restored after introducing

$$
\mathcal{D}_{\alpha}^{-}=u^{-a} \mathcal{D}_{\alpha a}=\left[\partial^{--}, \mathcal{D}_{\alpha}^{+}\right] .
$$


Then the remaining commutators are

$$
\left[\mathcal{D}_{\alpha}^{+}, \mathcal{D}_{\beta}^{-}\right]_{\rho}^{\rho^{\prime}}=\delta_{\rho}{ }^{\rho^{\prime}} \Omega_{\alpha \beta} R Z^{0}+\{\}_{(\alpha \beta) \rho}{ }^{\rho^{\prime}}, \quad\left[\mathcal{D}_{\alpha}^{-}, \mathcal{D}_{\beta}^{-}\right]_{\rho}^{\rho^{\prime}}=2 \delta_{\rho}{ }^{\rho^{\prime}} \Omega_{\alpha \beta} R Z^{--}
$$

One should also add the commutators between the harmonic derivatives (3.9) and the commutators of the latter with $\mathcal{D}_{\alpha}^{+}, \mathcal{D}_{\beta}^{-}$: they immediately follow from the definition of $\mathcal{D}_{\alpha}^{ \pm}$

The most important step is to single out the minimal closed set of operators which forms a Cauchi-Riemann (CR)-structure and is equivalent to the quaternionic geometry constraints (2.14). In the present case this set includes the following operators

$$
\left\{\mathcal{D}_{\alpha}^{+}, Z^{++}, Z^{0}, \mathcal{D}^{++}, \mathcal{D}^{0}, \mathcal{D}^{--}-Z^{--}\right\}
$$

Their algebra is given by

$$
\begin{gathered}
{\left[\mathcal{D}_{\alpha}^{+}, \mathcal{D}_{\beta}^{+}\right]=-2 \delta_{\rho}^{\rho^{\prime}} \Omega_{\alpha \beta} R Z^{++}} \\
{\left[\mathcal{D}^{++}, \mathcal{D}_{\alpha}^{+}\right]=0} \\
{\left[Z^{++}, \mathcal{D}_{\alpha}^{+}\right]=0, \quad\left[\mathcal{D}^{--}-Z^{--}, \mathcal{D}_{\alpha}^{+}\right]=0} \\
{\left[\mathcal{D}^{0}, \mathcal{D}_{\alpha}^{+}\right]=\left[Z^{0}, \mathcal{D}_{\alpha}^{+}\right]=\mathcal{D}_{\alpha}^{+}}
\end{gathered}
$$

and also

$$
\begin{gathered}
{\left[\mathcal{D}^{++}, Z^{++}\right]=0,\left[\mathcal{D}^{++}, \mathcal{D}^{0}\right]=-2 \mathcal{D}^{++}} \\
{\left[\mathcal{D}^{0}, Z^{++}\right]=2 Z^{++},\left[Z^{0}, \mathcal{D}^{++}\right]=2 Z^{++},\left[Z^{0}, Z^{++}\right]=2 Z^{++}} \\
{\left[\mathcal{D}^{--}-Z^{--}, \mathcal{D}^{0}\right]=-2\left(\mathcal{D}^{--}-Z^{--}\right),\left[\mathcal{D}^{--}-Z^{--}, Z^{0}\right]=0} \\
{\left[\mathcal{D}^{--}-Z^{--}, \mathcal{D}^{++}\right]=Z^{0}-\mathcal{D}^{0},\left[\mathcal{D}^{--}-Z^{--}, Z^{++}\right]=0}
\end{gathered}
$$

The relations (4.18) are written down for completeness, these are satisfied in the central basis by the definition of harmonic derivatives. The basic relations which actually underly the quaternionic geometry are those given in eqs. (4.14) - (4.17). To be convinced of that these relations indeed amount to the original constraint (2.14) one proceeds as follows.

First, eqs. (4.17) imply that $\mathcal{D}_{\alpha}^{+}$has coinciding $U(1)$ charge (the eigenvalue of $\mathcal{D}^{0}$ ) and $S p(1)$ weight (the eigenvalue of $Z^{0}$ ). So, its most general form consistent with (4.17) and the choice of $S p(n)$ as the tangent space group is given by (in the basis $\{z, w\}$ )

$$
\mathcal{D}_{\alpha}^{+}=E_{\alpha}^{+\mu k} \partial_{\mu k}+\omega_{\alpha(\rho \sigma)}^{+} \Gamma^{(\rho \sigma)}+\omega_{\alpha}^{-} Z^{++}+\omega_{\alpha}^{+} Z^{0}-\omega_{\alpha}^{+3} Z^{--}
$$


Here

$$
\begin{aligned}
Z^{0} E_{\alpha}^{+\mu k}=E_{\alpha}^{+\mu k} & \Rightarrow E_{\alpha}^{+\mu k}=z^{0} \tilde{E}_{\alpha}^{+\mu k} \\
Z^{0} \omega_{\alpha(\rho \sigma)}^{+}=\omega_{\alpha(\rho \sigma)}^{+} & \Rightarrow \omega_{\alpha(\rho \sigma)}^{+}=z^{0} \tilde{\omega}_{\alpha(\rho \sigma)}^{+} \\
Z^{0} \omega_{\alpha}^{-}=-\omega_{\alpha}^{-} & \Rightarrow \omega_{\alpha}^{-}=\left(z^{0}\right)^{-1} \tilde{\omega}_{\alpha}^{-} \\
Z^{0} \omega_{\alpha}^{+}=\omega_{\alpha}^{+} & \Rightarrow \omega_{\alpha}^{+}=z^{0} \tilde{\omega}_{\alpha}^{+} \\
Z^{0} \omega_{\alpha}^{+3}=3 \omega_{\alpha}^{+3} & \Rightarrow \omega_{\alpha}^{+3}=z^{0} \tilde{\omega}_{\alpha}^{+3}
\end{aligned}
$$

and the objects with tilde display no $z^{0}$ dependence. Then, exploiting (4.16) and the commutation relations between $Z$-operators, one completely fixes the $z$-dependence of these objects

$$
\begin{aligned}
\left(\mathcal{D}^{--}-Z^{--}\right) \tilde{E}_{\alpha}^{+\mu k}=Z^{++} \tilde{E}_{\alpha}^{+\mu k}=0 & \Rightarrow \tilde{E}_{\alpha}^{+\mu k}=\tilde{E}_{\alpha}^{+\mu k}(x, w) \\
\left(\mathcal{D}^{--}-Z^{--}\right) \tilde{\omega}_{\alpha(\rho \sigma)}^{+}=Z^{++} \tilde{\omega}_{\alpha(\rho \sigma)}^{+}=0 & \Rightarrow \tilde{\omega}_{\alpha(\rho \sigma)}^{+}=\tilde{\omega}_{\alpha(\rho \sigma)}^{+}(x, w) \\
\left(\mathcal{D}^{--}-Z^{--}\right) \tilde{\omega}_{\alpha}^{+3}=Z^{++} \tilde{\omega}_{\alpha}^{+3}=0 & \Rightarrow \tilde{\omega}_{\alpha}^{+3}=\tilde{\omega}_{\alpha}^{+3}(x, w) \\
\left(\mathcal{D}^{--}-Z^{--}\right) \tilde{\omega}_{\alpha}^{+}=0, \frac{\partial}{\partial z^{--}} \tilde{\omega}_{\alpha}^{+}=\tilde{\omega}_{\alpha}^{+3} & \Rightarrow \\
\tilde{\omega}_{\alpha}^{+}=\tilde{\omega}_{\alpha}^{+}(x, w) & +z^{--} \tilde{\omega}_{\alpha}^{+3}(x, w) \\
\left(\mathcal{D}^{--}-Z^{--}\right) \tilde{\omega}_{\alpha}^{-}=0, \frac{\partial}{\partial z^{--}} \tilde{\omega}_{\alpha}^{-}=2 \tilde{\omega}_{\alpha}^{+} & \Rightarrow \\
\tilde{\omega}_{\alpha}^{-}=\tilde{\omega}_{\alpha}^{-}(x, w)+2 z^{--} \tilde{\omega}_{\alpha}^{+}(x, w) & +\left(z^{--}\right)^{2} \tilde{\omega}_{\alpha}^{+3}(x, w) .
\end{aligned}
$$

Finally, by using (4.15), we fix the $w$-dependence of $\tilde{E}_{\alpha}^{+\mu k}, \tilde{\omega}_{\alpha(\rho \sigma)}^{+}, \tilde{\omega}_{\alpha}^{-}, \tilde{\omega}_{\alpha}^{+}$and $\tilde{\omega}_{\alpha}^{+3}$ as

$$
\begin{aligned}
\tilde{E}_{\alpha}^{+\mu k} & =w^{+i} e_{\alpha i}^{\mu k}(x), \tilde{\omega}_{\alpha(\rho \sigma)}^{+}=w^{+i} \omega_{\alpha i(\rho \sigma)}(x) \\
\tilde{\omega}_{\alpha}^{+3} & =-w^{+i} w^{+j} w^{+k} \omega_{\alpha(i j k)}(x) \\
\tilde{\omega}_{\alpha}^{+} & =-w^{+i} \omega_{\alpha i}(x)-w^{+i} w^{+j} w^{-k} \omega_{\alpha(i j k)}(x) \\
\tilde{\omega}_{\alpha}^{-} & =-2 w^{-i} \omega_{\alpha i}(x)-w^{+i} w^{-j} w^{-k} \omega_{\alpha(i j k)}(x),
\end{aligned}
$$

where the sign "-" was chosen for further convenience. Combining $\omega_{\alpha i}(x)$ and $\omega_{\alpha(i j k)}(x)$ into the single object

$$
\omega_{\alpha i(j k)}(x) \equiv \omega_{\alpha(i j k)}(x)+\epsilon_{i j} \omega_{\alpha k}(x)+\epsilon_{i k} \omega_{\alpha j}(x),
$$

we eventually represent $\mathcal{D}_{\alpha}^{+}(4.19)$ as

$$
\begin{aligned}
\mathcal{D}_{\alpha}^{+} & =E_{\alpha}^{+\mu k} \partial_{\mu k}-\omega_{\alpha(\rho \sigma)}^{+} \Gamma^{(\rho \sigma)}+\omega_{\alpha}^{+3} \partial_{w}^{--}-\omega_{\alpha}^{+-+} Z^{0} \\
& -\left(\omega_{\alpha}^{+--} \frac{\partial}{\partial z^{--}}+2 \omega_{\alpha}^{+-+} z^{--} \frac{\partial}{\partial z^{--}}+\omega_{\alpha}^{+3} \frac{\partial}{\partial z^{++}}\right) \\
& \equiv \tilde{\mathcal{D}}_{\alpha}^{+}-z^{0}\left(\tilde{\omega}_{\alpha}^{+--} \frac{\partial}{\partial z^{--}}+2 \tilde{\omega}_{\alpha}^{+-+} z^{--} \frac{\partial}{\partial z^{--}}+\tilde{\omega}_{\alpha}^{+3} \frac{\partial}{\partial z^{++}}\right) \\
\tilde{\mathcal{D}}_{\alpha}^{+} & =z^{0} \tilde{\nabla}_{\alpha}^{+}-z^{0} \tilde{\omega}_{\alpha}^{+-+} Z^{0} \\
& \equiv z^{0}\left(\tilde{\Delta}_{\alpha}^{+}-\tilde{\omega}^{+} \Gamma+\tilde{\omega}^{+3} \partial_{w}^{--}\right)-z^{0} \tilde{\omega}_{\alpha}^{+-+} Z^{0}
\end{aligned}
$$




$$
\left[\tilde{\mathcal{D}}_{\alpha}^{+}, \tilde{\mathcal{D}}_{\beta}^{+}\right]=0
$$

Here

$$
\begin{aligned}
\tilde{\Delta}_{\alpha}^{+} & =\tilde{E}_{\alpha}^{+\mu k} \partial_{\mu k}=w^{+i} e_{\alpha i}^{\mu k}(x) \partial_{\mu k} \\
\tilde{\omega}_{\alpha(\rho \sigma)}^{+} & =w^{+i} \omega_{\alpha i(\rho \sigma)}(x) \\
\tilde{\omega}_{\alpha}^{+-+} & =w^{+i} w^{-j} w^{+k} \omega_{\alpha i(j k)}(x) \\
\tilde{\omega}_{\alpha}^{+--} & =w^{+i} w^{-j} w^{-k} \omega_{\alpha i(j k)}(x) \\
\tilde{\omega}_{\alpha}^{+3} & =w^{+i} w^{+j} w^{k} \omega_{\alpha i(j k)}(x) .
\end{aligned}
$$

Now, returning to the basis $\left\{v_{a}^{i}, u^{ \pm a}\right\}$, it is easy to show that

$$
\mathcal{D}_{\alpha}^{+}=u^{+a} \mathcal{D}_{\alpha a}
$$

where $\mathcal{D}_{\alpha a}$ is given by eq.(4.2). Finally, substituting (4.26) into eq.(4.14) and keeping in mind that $Z^{++}=-\frac{1}{2} u^{+a} u^{+b} Z_{a b}$, one arrives at the relation (4.5) which, as we have seen before, is the same as the original constraint (2.14).

Two comments are in order here.

Firstly, it is just the equality of the $U(1)$ charge and the $S p(1)$ weight of $\mathcal{D}_{\alpha}^{+}$(eqs. (4.17) ) that guarantees the compatibility of the constraints (4.15) and the second of the constraints (4.16). Commuting $\mathcal{D}^{++}$with the l.h.s. of the latter equation one gets

$$
\left[\mathcal{D}^{0}-Z^{0}, \mathcal{D}_{\alpha}^{+}\right]=0
$$

that is fulfilled as a consequence of eqs. (4.17).

Secondly, it should be stressed that the $S U(2)$ algebra for $Z_{a b}$ immediately stems from the important relations contained in the set of the harmonic constraints (4.18)

$$
\left[\mathcal{D}^{++}, Z^{++}\right]=0,\left[\mathcal{D}^{++}, Z^{0}\right]=-2 Z^{++},\left[Z^{0}, Z^{++}\right]=2 Z^{++} .
$$

First of these equations implies $Z^{++}=-\frac{1}{2} u^{+a} u^{+b} Z_{a b}$, then from the second one it follows $Z^{0}=-u^{+a} u^{-b} Z_{a b}$ and, finally, substituting these expressions for $Z^{0}$ and $Z^{++}$into the third equation and using $u_{a}^{ \pm}$algebra, one deduces the $S p(1)$ commutation relations (3.4).

To summarize, we have demonstrated that the defining constraints of the quaternionic geometry (2.14) in the space $\left\{x^{\mu k}\right\}$ amount to the constraints (4.14)-(4.17) in the bi-harmonic space $\left\{x^{\mu k}, v^{ \pm i}, u^{ \pm a}\right\}=\left\{x^{\mu k}, z, w\right\}$. Like in all other cases to which the harmonic analyticity is relevant, the property that the operators $\left\{\mathcal{D}_{\alpha}^{+}, Z^{++}, \mathcal{D}^{0}, Z^{0}, \mathcal{D}^{--}-\right.$ $Z^{--}$\} form a closed algebra amounts to the possibility to define covariantly analytic fields. This time, they are defined by the following analyticity conditions

$$
\begin{aligned}
\mathcal{D}_{\alpha}^{+} \Phi^{(p, q)}(x, v, u) & =0 \\
Z^{++} \Phi^{(p, q)}(x, v, u) & =0 \\
\left(\mathcal{D}^{--}-Z^{--}\right) \Phi^{(p, q)}(x, v, u) & =0 \\
Z^{0} \Phi^{(p, q)}(x, v, u) & =p \Phi^{(p, q)}(x, v, u) \\
\mathcal{D}^{0} \Phi^{(p, q)}(x, v, u) & =q \Phi^{(p, q)}(x, v, u) .
\end{aligned}
$$


Eq. (4.30) combined with the relations $\left[\mathcal{D}^{++}, Z^{++}\right]=0,\left[\mathcal{D}^{++}, Z^{0}\right]=-2 Z^{++}$imply that the harmonic derivative $\mathcal{D}^{++}$preserves this analyticity provided $p=q: \mathcal{D}^{++} \Phi^{(q, q)}$ is again an analytic field with the $U(1)$ charge $q+2$ and the $S p(1)$ weight $q$. Recall that the original tensor fields with the transformation law (2.1) form a subclass in the variety of bi-harmonic fields $\Phi^{(q, q)}$.

Note that the conditions $(4.30)$ - $(4.33)$ mean that the field $\Phi^{(p, q)}$ does not depend on the coordinates $z^{++}, z^{--}$and involves the factorized $z^{0}$ dependence. So, one is eventually left with the following harmonic analyticity condition (see definition (4.24))

$$
\left(\tilde{\nabla}_{\alpha}^{+}-p \tilde{\omega}_{\alpha}^{+-+}\right) \tilde{\Phi}^{(p, q)}\left(x, w^{ \pm}\right)=0, \quad \Phi^{(p, q)}(x, v, u)=\left(z^{0}\right)^{p} \tilde{\Phi}^{(p, q)}(x, w) .
$$

It implies, like in the HK case [2], the existence of an invariant analytic subspace $\left\{x_{A}^{+}, w_{A}^{ \pm}\right\}$in $\left\{x^{\mu i}, w^{ \pm}\right\}$. The principal difference from the HK case is that the harmonics $w^{ \pm}$(and their analytic basis counterparts $w_{A}^{ \pm}$) have non-trivial transformation properties under the tangent space local $S p(1)$ group (correspondingly, $\tilde{\nabla}_{\alpha}^{+}$in eq. (4.34) contains a differentiation with respect to $w$ ). This peculiarity of the quaternionic case will entail, in particular, the necessity of an additional bridge while passing to the analytic world, namely the one relating the $\tau$ - and $\lambda$ - basis $w$ harmonics (see below).

For our further purposes it will be crucial that the analyticity (4.29) - 4.33) implies, like in the HK and self-dual Yang-Mills cases [1, 2], the existence of some basis and frame (the $\lambda$-world) where this analyticity is manifest. Just as in these cases, $\lambda$-world turns out to be most appropriate for solving the integrability conditions (4.14 - (4.17), (4.18). On all stages of computation we will keep the derivatives with respect to $z$-coordinates. This guarantees that we will not lose any information encoded in eqs. (4.14) - (4.17), (4.18).

\section{$5 \quad$ Bridges to $\lambda$-world}

As was just mentioned, the existence of the CR-structure (4.14) - 4.17), (4.18) implies possiblity to pass to the basis and frame called $\lambda$-world where the analyticity associated with this structure becomes manifest. This means that differential operators forming the CR-structure are reduced in the $\lambda$-world to partial derivatives.

In principal, there are different choices of the change of the coordinates $\left\{x^{\mu i}, z^{(a b)}, w^{ \pm}\right\}$ leading to the analytic basis. The minimal and most convenient option is as follows

$$
\begin{aligned}
z_{A}^{++} & =z^{++}+v^{++}(x, w) \\
z_{A}^{--} & =t^{2}(x, w)\left[z^{--}+v^{--}(x, w)\right] \\
z_{A}^{0} & =t(x, w) z^{0} \\
w_{A}^{-i} & =w^{-i} \\
w_{A}^{+i} & =w^{+i}-+v^{++}(x, w) w^{-i} \\
x_{A}^{ \pm \mu} & =x^{\mu i} w_{i}^{ \pm}+v^{ \pm \mu}(x, w) .
\end{aligned}
$$

The bridges can be consistently chosen independent of the coordinates $z$ in view of the bi-harmonic integrability conditions (4.18). Then the basic integrability condition (4.14) 
allows one to define the bridges by the following constraints (analogous to those employed in the HK case [2])

$$
\begin{aligned}
\mathcal{D}_{\alpha}^{+} z_{A}^{++}=0 & \Rightarrow \tilde{\Delta}_{\alpha}^{+} v^{++}-\left(1-\partial_{w}^{--} v^{++}\right) \tilde{\omega}_{\alpha}^{+3}=0 \\
\mathcal{D}_{\alpha}^{+} z^{0}=0 & \Rightarrow \tilde{\Delta}_{\alpha}^{+} t+\tilde{\omega}_{\alpha}^{+3} \partial_{w}^{--} t-\tilde{\omega}_{\alpha}^{+-+} t=0 \\
\mathcal{D}_{\alpha}^{+} w_{A}^{+i} & =0, \quad \mathcal{D}_{\alpha}^{+} w_{A}^{-i}=0, \quad \mathcal{D}_{\alpha}^{+} x_{A}^{+\mu}=0
\end{aligned}
$$

where $\mathcal{D}_{\alpha}^{+}$and $\tilde{\Delta}_{\alpha}^{+}$were defined in eqs. 4.23 - (4.25). Note that first of eqs. (5.5) is automatically satisfied as a consequence of (5.3) (the second one is satisfied trivially). The definition (5.3) - (5.5) admits the pregauge group with analytic parameters

$$
\begin{gathered}
\delta z_{A}^{++}=\lambda^{++}, \quad \delta z_{A}^{0}=\lambda z_{A}^{0}, \quad \delta w_{A}^{-i}=0, \quad \delta w_{A}^{+i}=-\lambda^{++} w_{A}^{-i}, \quad \delta x_{A}^{+\mu}=\lambda^{+\mu} \\
\mathcal{D}_{\alpha}^{+} \lambda^{++}=\mathcal{D}_{\alpha}^{+} \lambda=\mathcal{D}_{\alpha}^{+} \lambda^{+\mu}=0,
\end{gathered}
$$

while the remaining coordinates transform with general (though $z$-independent) parameters $\lambda^{--}, \lambda^{-\mu}$

$$
\delta z_{A}^{--}=\lambda^{--}+2 \lambda z_{A}^{--}, \quad \delta x_{A}^{-\mu}=\lambda^{-\mu} .
$$

From eqs. (5.1), (5.2), (5.6), (5.8) one finds the transformations of bridges

$$
\begin{aligned}
\delta v^{++} & =\lambda^{++}-\tau^{++}, \quad \delta v^{--}=2 \tau^{+-} v^{--}-\tau^{--}+t^{-2} \lambda^{--} \\
\delta v^{+\mu} & =\lambda^{+\mu}-w^{+i} \tau^{\mu i}(x)-x^{\mu i} \tau^{++} w^{-i}, \quad \delta v^{-\mu}=\lambda^{-\mu}-w^{-i} \tau^{\mu i}(x) .
\end{aligned}
$$

Finally, we should pass to the analytic $S p(n)$ frame by rotating all tangent space $S p(n)$ indices by an appropriate matrix bridge. In this new frame the tangent space group $S p(n)$ is realized by gauge transformations with analytic parameters. Actually, this procedure is the same as in the HK case [2], so we do not give here details of it.

Performing the change of variables (5.1), (5.2) in the expressions (3.16), (3.17) and passing to the analytic $S p(n)$ frame, we find the $\lambda$-world form of harmonic and $z$ derivatives

$$
\begin{aligned}
\mathcal{D}^{++}= & \mathcal{D}_{\lambda}^{++}+\phi^{++}\left(z_{A}^{0} \frac{\partial}{\partial z_{A}^{0}}\right)-\left[H^{+4}-\left(z_{A}^{++}\right)^{2}\right] \frac{\partial}{\partial z_{A}^{++}} \\
& +\left[2 \phi^{++} z_{A}^{--}+\left(z_{A}^{0}\right)^{2}+\phi\right] \frac{\partial}{\partial z_{A}^{--}}+z_{A}^{++}\left(z_{A}^{0} \frac{\partial}{\partial z_{A}^{0}}-\mathcal{D}^{0}\right) \\
\mathcal{D}_{\lambda}^{++} \equiv & \Delta^{++}+\omega^{++}=\partial_{A}^{++}+H^{+3 \mu} \partial_{\mu}^{-}+H^{++-\mu} \partial_{\mu}^{+}+H^{+4} \partial_{A}^{--}+\omega^{++} \\
\mathcal{D}^{0}= & \partial_{A}^{0}+\left(x_{A}^{+\mu} \partial_{\mu}^{-}-x_{A}^{-\mu} \partial_{\mu}^{+}+2 z_{A}^{++} \frac{\partial}{\partial z_{A}^{++}}-2 z_{A}^{--} \frac{\partial}{\partial z_{A}^{--}}\right) \\
\mathcal{D}^{--}= & \left(z_{A}^{0}\right)^{-2}\left\{\psi \mathcal{D}_{\lambda}^{--}+\left[\left(z_{A}^{0}\right)^{2}-\psi\right] \frac{\partial}{\partial z_{A}^{++}}+\left(\phi^{--}+z_{A}^{--}\right) z_{A}^{0} \frac{\partial}{\partial z_{A}^{0}}\right. \\
& \left.+\left[2 \phi^{--} z_{A}^{--}+H^{-4}+\left(z_{A}^{--}\right)^{2}\right] \frac{\partial}{\partial z_{A}^{--}}\right\} \\
\equiv & \Delta^{--}+\omega^{--}=\partial_{A}^{--}+H^{--+\mu} \partial_{\mu}^{-}+H^{-3 \mu} \partial_{\mu}^{+}+\omega^{--} \\
\mathcal{D}_{\lambda}^{--} & \left(z_{A}^{0}\right)^{2} \frac{\partial}{\partial z_{A}^{--}} \\
Z^{++} &
\end{aligned}
$$




$$
\begin{aligned}
Z^{--} & =\mathcal{D}^{--}-\left(z_{A}^{0}\right)^{2} \frac{\partial}{\partial z_{A}^{++}} \\
Z^{0} & =z_{A}^{0} \frac{\partial}{\partial z_{A}^{0}} .
\end{aligned}
$$

Here $\partial_{\mu}^{+}, \partial_{\mu}^{-}$stand for partial derivatives with respect to the analytic basis coordinates $x_{A}^{-\mu}, x_{A}^{+\mu}$, the objects $\omega^{++}, \omega^{--}$are the harmonic $S p(n)$ connections arising as a result of passing to the analytic frame, and the remaining quantities (vielbeins) are defined by

$$
\begin{aligned}
H^{+3 \mu} & =\partial^{++} v^{+\mu}+v^{++} x_{A}^{+\mu} \\
H^{++-} \mu & =\partial^{++} v^{-\mu}-v^{+\mu}+x_{A}^{+\mu}-v^{++} x_{A}^{-\mu} \\
H^{+4} & =-\left[\partial^{++} v^{++}+\left(v^{++}\right)^{2}\right] \\
\phi^{++} & =\partial^{++} \ln t-v^{++} \\
\phi & =t^{2}\left(\partial^{++} v^{--}-1\right) \\
\psi & =t^{2}\left(1-\partial^{--} v^{++}\right) \\
H^{--}+\mu & =\frac{1}{1-\partial^{--} v^{++}} \partial^{--} x_{A}^{ \pm \mu}=\Delta^{--} x_{A}^{ \pm \mu} \\
\Delta^{--} & =\frac{1}{1-\partial^{--} v^{++}} \partial^{--} \\
\phi^{--} & =t^{2}\left(\partial^{--} \ln t-v^{--}\right) \\
H^{-4} & =t^{4}\left[\partial^{--} v^{--}+\left(v^{--}\right)^{2}\right] .
\end{aligned}
$$

The vielbeins $(5.17)-(5.18)$ transform as follows

$$
\begin{aligned}
\delta H^{+3 \mu} & =\Delta^{++} \lambda^{+\mu}+\lambda^{++} x_{A}^{+\mu} \\
\delta H^{++-} & =\Delta^{++} \lambda^{-\mu}-\lambda^{++} x_{A}^{-\mu} \\
\delta H^{+4} & =-\Delta^{++} \lambda^{++} \\
\delta \phi^{++} & =\Delta^{++} \lambda-\lambda^{++} \\
\delta \phi & =\Delta^{++} \lambda^{--}-2 \phi^{++} \lambda^{--}+2 \lambda \phi \\
\delta \psi & =\left(2 \lambda-\Delta^{--} \lambda^{++}\right) \psi \\
\delta H^{--+\mu} & =\Delta^{--} \lambda^{+\mu}+\left(\Delta^{--} \lambda^{++}\right) H^{--+\mu} \\
\delta H^{-3 \mu} & =\Delta^{--} \lambda^{-\mu}+\left(\Delta^{--} \lambda^{++}\right) H^{-3 \mu} \\
\delta \phi^{--} & =2 \lambda \phi^{--}-\lambda^{--}+\left(\Delta^{--} \lambda\right) \psi \\
\delta H^{-4} & =4 \lambda H^{-4}-2 \phi^{--} \lambda^{--}-\left(\Delta^{--} \lambda^{--}\right) \psi .
\end{aligned}
$$

At this step it is convenient to choose the following gauges

$$
\begin{gathered}
H^{++-\mu}=x_{A}^{+\mu} \Rightarrow \partial^{++} v^{-\mu}-v^{+\mu}-v^{++} x_{A}^{-\mu}=0, \quad \lambda^{+\mu}=\Delta^{++} \lambda^{-\mu}-\lambda^{++} x_{A}^{-\mu} \\
\phi^{--}=0 \Rightarrow v^{--}=\partial^{--} \ln t, \quad \lambda^{--}=\psi \Delta^{--} \lambda .
\end{gathered}
$$


In the gauge (5.22) the expression (5.12) and the last ones of the expressions (5.17) and (5.18) are somewhat simplified

$$
\begin{gathered}
\mathcal{D}^{--}=\left(z_{A}^{0}\right)^{-2}\left\{\psi \mathcal{D}_{\lambda}^{--}+\left[\left(z_{A}^{0}\right)^{2}-\psi\right] \frac{\partial}{\partial z_{A}^{++}}+z_{A}^{--}\left(z_{A}^{0} \frac{\partial}{\partial z_{A}^{0}}\right)+\left[H^{-4}+\left(z_{A}^{--}\right)^{2}\right] \frac{\partial}{\partial z_{A}^{--}}\right\}(5 \\
\phi=-\psi\left(1-\Delta^{--} \phi^{++}\right) \\
H^{-4}=t^{3}\left(\partial^{--}\right)^{2} t
\end{gathered}
$$

The specific feature of the case under consideration compared to the HK one is the presence of new harmonic vielbeins $\phi^{++}$and $\phi^{--}$which multiply the $S p(1)$ weight operator $z_{A}^{0} \partial / \partial z_{A}^{0}$ in $\mathcal{D}^{++}$and $\mathcal{D}^{--}$. When the latter are applied to the functions having $p=q$ and bearing no dependence on $z_{A}^{++}, z_{A}^{--}$, the new vielbeins can be interpreted as some harmonic $S p(1)$ connections. For instance, on such functions $\mathcal{D}^{++}$takes the following form

$$
\mathcal{D}^{++}=\mathcal{D}_{\lambda}^{++}+\phi^{++} \mathcal{D}^{0}
$$

and so it preserves the analytic subspace $\left\{x^{+\mu}, w_{A}^{ \pm i}\right\}$ (as will be clear soon, $\phi^{++}$and the vielbeins entering $\mathcal{D}_{\lambda}^{++}$are analytic).

It is instructive to give here how the $z$-independent parts of the derivatives $\mathcal{D}^{++}$and $\mathcal{D}^{--}$look in the original $\tau$-basis $\left\{x^{\mu i}, w^{ \pm i}\right\}$ :

$$
\Delta^{--}=\frac{1}{1-\partial_{w}^{--} v^{++}} \partial_{w}^{--}, \quad \Delta^{++}=\partial_{w}^{--}+v^{++} D^{0} \|
$$

where the slash means the part of $D^{0}$ containig no $z$-derivatives. They are transformed under the $\lambda$-world gauge group in the following way (once again, when act on the functions independent of $z_{A}^{++}, z_{A}^{--}$and possessing $p=q$ )

$$
\delta \Delta^{++}=\lambda^{++} D^{0}\left\|, \quad \delta \Delta^{--}=\left(\Delta^{--} \lambda^{++}\right) \Delta^{--}-\Delta^{--} \lambda D^{0}\right\|
$$

Note that the role of vielbein $\psi$ in eqs. (5.12) or (5.23) consists in converting the $S p(1)$ weight transformation with the nonanalytic parameter $\Delta_{\lambda}^{++} \lambda^{++}$into a transformation with the analytic parameter $\lambda$.

Finally, we quote here the general form of $\mathcal{D}_{\alpha}^{+}$in the $\lambda$-world. Taking into account the defining relations (5.3) - (5.5) and the property that this derivative does not contain $S p(n)$ connection after passing to the $\lambda$-frame (cf. the similar situation in the HK case [2]), it is given by

$$
\mathcal{D}_{A \alpha}^{+}=E_{\alpha}^{\mu} \partial_{\mu}^{+}+\rho_{\alpha}^{-} \frac{\partial}{\partial z_{A}^{--}} \equiv \Delta_{\alpha}^{+}+\rho_{\alpha}^{-} \frac{\partial}{\partial z_{A}^{--}}=\left(z_{A}^{0}\right)\left(\tilde{\Delta}_{\alpha}^{+}+\tilde{\rho}_{\alpha}^{-} \frac{\partial}{\partial z_{A}^{--}}\right) .
$$

The transformation properties of this important object and the equations for the vielbeins $E_{\alpha}^{\mu}$ and $\rho_{\alpha}^{-}$following from the quaternionic geometry constraints (4.14) - (4.17), (4.18) will be discussed in the next section. 


\section{$6 \lambda$-world geometry: consequences of constraints}

Our ultimate purpose will be to draw all the consequences the defining constraints (4.14)(4.17), 4.18) entail for various vielbeins and connections in the $\lambda$-world and to express (in the next Section) the latter in terms of a few unconstrained prepotentials. As we will see below, these prepotentials coincide with certain vielbeins multiplying the derivatives with respect to $z$-coordinates. This automatic appearance of the prepotentials as the objects having a clear geometrical meaning is the actual advantage of dealing with the $z$-extension of the quaternionic manifolds.

Recall that the HK geometry prepotentials [2] can be deduced in two equivalent ways: either as some new objects in the process of solving the HK constraints or as the vielbeins associated with an extension of the original HK manifold by a kind of "central charge" coordinates. In the quaternionic case just the second, most geometric approach proves to be adequate, because, as we saw in previous Sections, it is necessary to extend the initial manifold by $z$-coordinates for giving the CR-structure interpretation to the quaternionic geometry constraints.

\subsection{Derivative $\mathcal{D}_{A \alpha}^{+}$}

We begin with studying the covariant derivative $\mathcal{D}_{A \alpha}^{+}$defined in eq. (5.29). The vielbeins $E_{\alpha}^{\mu}$ and $\rho_{\alpha}^{-}$transform under the analytic tangent space transformations and diffeomorphisms (5.8) according to

$$
\begin{aligned}
\delta E_{\alpha}^{\mu} & =\lambda_{\alpha}{ }^{\beta} E_{\beta}^{\mu}+E_{\alpha}^{\nu} \partial_{\nu}^{+} \lambda^{-\mu} \\
\delta \rho_{\alpha}^{-} & =2 \lambda \rho_{\alpha}^{-}+E_{\alpha}^{\mu} \partial_{\mu}^{+} \lambda^{--}+\lambda_{\alpha}{ }^{\beta} \rho_{\beta}^{-} .
\end{aligned}
$$

One should remember that $\mathcal{D}_{A \alpha}^{+}$has the $S p(1)$ weght 1 and hence it is linear in the coordinate $z_{A}^{0}$. The $z$-independent vielbeins $\tilde{E}_{\alpha}^{\mu}=\left(z_{A}^{0}\right)^{-1} E_{\alpha}^{\mu}$ and $\tilde{\rho}_{\alpha}^{-}=\left(z_{A}^{0}\right)^{-1} \rho_{\alpha}^{-}$transform under the $S p(1) \lambda$ - transformations as

$$
\delta \tilde{E}_{\alpha}^{\mu}=-\lambda \tilde{E}_{\alpha}^{\mu}, \quad \delta \tilde{\rho}_{\alpha}^{-}=\lambda \tilde{\rho}_{\alpha}^{-} .
$$

The basic constraint (4.14)

$$
\left[\mathcal{D}_{A \alpha}^{+}, \mathcal{D}_{A \beta}^{+}\right]=-2 \Omega_{\alpha \beta} R\left(z_{A}^{0}\right)^{2} \frac{\partial}{\partial z^{--}}
$$

implies the following relations

$$
\begin{gathered}
E_{[\alpha}^{\mu} \partial_{A \mu}^{+} E_{\beta]}^{\rho}=0 \\
\Delta_{[\alpha}^{+} \rho_{\beta]}^{-}=-\Omega_{\alpha \beta} R\left(z_{A}^{0}\right)^{2}, \quad \text { or } \quad \tilde{\Delta}_{[\alpha}^{+} \rho_{\beta]}^{-}=-\Omega_{\alpha \beta} R .
\end{gathered}
$$

Eq. 6.5) is the same as in the HK case while (6.6) is new.

The next important constraint is

$$
\left[\mathcal{D}_{A \alpha}^{+}, \mathcal{D}^{++}\right]=0
$$


It implies the analyticity of the vielbeins and connections entering into $\mathcal{D}^{++}$

$$
\Delta_{\alpha}^{+} H^{+3 \mu}=\Delta_{\alpha}^{+} H^{+4}=\Delta_{\alpha}^{+} \omega^{++}=\Delta_{\alpha}^{+} \phi^{++}=0
$$

and also results in the relation

$$
\mathcal{D}_{\lambda}^{++} E_{\alpha}^{\mu}+\phi^{++} E_{\alpha}^{\mu}=0,
$$

or, equivalently,

$$
\begin{gathered}
E_{(\alpha}^{\mu} \Delta^{++} E_{\mu \beta)}^{-1}=\omega_{\alpha \beta}^{++} \\
E_{[\alpha}^{\mu} \Delta^{++} E_{\mu \beta]}^{-1}=\Omega_{\beta \alpha} \phi^{++},
\end{gathered}
$$

and in the following one

$$
\mathcal{D}_{\lambda}^{++} \rho_{\alpha}^{-}-\phi^{++} \rho_{\alpha}^{-}-\Delta_{\alpha}^{+} \phi=0 .
$$

Note the appearance of a non-zero right-hand side in eq. (6.11) in contrast to an analogous equation in the HK case. The constraints (6.5), (6.6), (6.11), (6.12) will be solved later on, after defining the derivative $\mathcal{D}_{A \alpha}^{-}$.

\subsection{Consequences of the algebra of harmonic derivatives}

Like in the HK case, the vielbeins and connections entering the harmonic derivative $\mathcal{D}^{--}$ can be related to the analytic vielbeins (6.8) by the equation

$$
\left[\mathcal{D}^{++}, \mathcal{D}^{--}\right]=\mathcal{D}^{0} .
$$

Comparing the coefficients of the same derivatives in both sides of (6.13), one finds

$$
\begin{aligned}
\Delta^{--} H^{+4}=\Delta^{++} \ln \psi-2 \phi^{++} & \\
\Delta^{++} H^{-4}-4 \phi^{++} H^{-4}-\psi \Delta^{--} \phi & =0 \\
\left(\Delta^{++}+\Delta^{--} H^{+4}\right) H^{--+\mu}-\Delta^{--} H^{+3 \mu} & =x_{A}^{+\mu} \\
\left(\Delta^{++}+\Delta^{--} H^{+4}\right) H^{-3 \mu}-H^{--+\mu} & =x_{A}^{-\mu} \\
\left(\Delta^{++}+\Delta^{--} H^{+4}\right) \omega^{--}-\Delta^{--} \omega^{++}+\left[\omega^{++}, \omega^{--}\right] & =0 .
\end{aligned}
$$

Using eqs. (6.14) - (6.16) one may, at least iteratively, express $H^{--+\mu}, H^{-3 \mu}, \omega^{--}, \psi, H^{-4}$ in terms of $H^{+3 \mu}, H^{+4}, \phi^{++}$and $\omega^{++}$. Note the useful relation

$$
\left[\mathcal{D}_{\lambda}^{++}, \mathcal{D}_{\lambda}^{--}\right]=\mathcal{D}_{\lambda}^{0}-\left(\Delta^{--} H^{+4}\right) \mathcal{D}_{\lambda}^{--},
$$

which follows from eqs. (6.16). 


\subsection{Derivative $\mathcal{D}_{\alpha}^{-}$}

We define the $\lambda$-world derivative $\mathcal{D}_{\alpha}^{-}$on the pattern already employed in the $\tau$-world (eq. (4.11)

$$
\begin{aligned}
\mathcal{D}_{\alpha}^{-}=\left[\mathcal{D}^{--}, \mathcal{D}_{\alpha}^{+}\right]= & \left(z_{A}^{0}\right)^{-2}\left\{\left(E_{\alpha}^{-2 \mu}+z_{A}^{--} E_{\alpha}^{\mu}\right) \partial_{\mu}^{+}+E_{\alpha}^{-+\mu} \partial_{\mu}^{-}+E_{\alpha}^{+}\left(\partial_{A}^{--}-\frac{\partial}{\partial z_{A}^{++}}\right)\right. \\
& \left.+\left(E_{\alpha}^{-3}-z_{A}^{--} \rho_{\alpha}^{-}\right) \frac{\partial}{\partial z_{A}^{--}}-\rho_{\alpha}^{-}\left(z_{A}^{0} \frac{\partial}{\partial z_{A}^{0}}\right)+\omega_{\alpha}^{-}\right\}
\end{aligned}
$$

where

$$
\begin{aligned}
E_{\alpha}^{-2 \mu} & =\psi \mathcal{D}_{\lambda}^{--} E_{\alpha}^{\mu}-\Delta_{\alpha}^{+} \psi H^{-3 \mu}-\psi \Delta_{\alpha}^{+} H^{-3 \mu} \equiv z_{A}^{0} \tilde{E}_{\alpha}^{-2 \mu} \\
E_{\alpha}^{-+\mu} & =-\left(\Delta_{\alpha}^{+} \psi H^{--+\mu}+\psi \Delta_{\alpha}^{+} H^{--+\mu}\right) \equiv e_{\alpha}{ }^{\mu}=z_{A}^{0} \tilde{e}_{\alpha}{ }^{\mu} \\
E_{\alpha}^{+} & =-\Delta_{\alpha}^{+} \psi \equiv-\mathcal{L}_{\alpha}^{+}=-z_{A}^{0} \tilde{\mathcal{L}}_{\alpha}^{+} \\
E_{\alpha}^{-3} & =\psi \mathcal{D}_{\lambda}^{--} \rho_{\alpha}^{-}-\Delta_{\alpha}^{+} H^{-4} \equiv z_{A}^{0} \tilde{E}_{\alpha}^{-3} \\
\omega_{\alpha}^{-} & =-\psi\left(\Delta_{\alpha}^{+} \ln \psi \omega^{--}+\Delta_{\alpha}^{+} \omega^{--}\right) \equiv z_{A}^{0} \tilde{\omega}_{\alpha}^{-} .
\end{aligned}
$$

A number of important constraints on these objects follows from considering the commutator

$$
\left[\mathcal{D}_{\alpha}^{+}, \mathcal{D}_{\beta}^{-}\right]_{\rho}^{\rho^{\prime}}=\delta_{\rho} \rho^{\prime} \Omega_{\alpha \beta} R Z^{0}+\left(R_{\alpha \beta}^{+-}\right)_{\rho}^{\rho^{\prime}}
$$

The vanishing of the torsion components $T_{\alpha \beta}^{+-(+\mu)}$ and $T_{\alpha \beta}^{+-(+2)}$ in the r.h.s. of (6.24) ( the coefficients of $\partial_{\mu}^{-}$and $\partial^{--}$) implies, respectively,

$$
\Delta_{\alpha}^{+} e_{\beta}^{\mu}=0, \quad \Delta_{\alpha}^{+} \mathcal{L}_{\beta}^{+}=0,
$$

i.e., $e_{\beta}^{\mu}$ and $\mathcal{L}_{\beta}^{+}$are analytic. Equating the coefficients of $Z^{0}$ in both sides of (6.24) and taking account of eq.(6.6), one finds

$$
\Delta_{(\alpha}^{+} \rho_{\beta)}^{-}=0
$$

Also, it is easy to evaluate the $S p(n)$ curvature $R_{\alpha \beta}^{+-}$

$$
\left(R_{\alpha \beta}^{+-}\right)_{\rho}{ }^{\rho^{\prime}}=\left(\tilde{\Delta}_{\alpha}^{+} \tilde{\omega}_{\beta}^{-}\right)_{\rho}^{\rho^{\prime}}=\left\{\psi^{-1} \tilde{\mathcal{L}}_{\alpha}^{+} \tilde{\omega}_{\beta}^{-}+\psi \tilde{\Delta}_{\alpha}^{+}\left(\psi^{-1} \tilde{\omega}_{\beta}^{-}\right)\right\}_{\rho}^{\rho^{\prime}}
$$

where, as before, tilde means that the relevant quantity does not contain the dependence on $z_{A}^{0}$. Using the explicit expression for $\tilde{\omega}_{\beta}^{-}$(6.23) one may easily be convinced that $R_{\alpha \beta}^{+-}$ is symmetric in the indices $\alpha, \beta$

$$
R_{[\alpha \beta]}^{+-}=\tilde{\Delta}_{[\alpha}^{+} \tilde{\omega}_{\beta]}^{-}=0 .
$$

There is also a number of additional relations following from eq.(6.24), but all these can be checked to be consequences of a few essential ones some of which have been already given and others will be presented below. 


\subsection{Vielbein $E_{\alpha}^{\mu}$}

Using eq. (6.20), one may express the vielbein $E_{\alpha}^{\mu}$ defined in eq. (5.29) through the analytic quantities $e_{\alpha}^{\mu}, \mathcal{L}_{\alpha}^{+}$and the harmonic vielbeins $\psi$ and $H^{--+\mu}$ :

$$
E_{\alpha}^{\mu}=\psi^{-1} A_{\alpha}^{\nu}\left(\partial H^{-1}\right)_{\nu}^{\mu}
$$

Here

$$
\begin{aligned}
A_{\alpha}{ }^{\nu} & \equiv e_{\alpha}{ }^{\nu}-\mathcal{L}_{\alpha}^{+} H^{--+\nu} \\
(\partial H)_{\nu}{ }^{\mu} & \equiv \partial_{\nu}^{+} H^{--+\mu}
\end{aligned}
$$

After some simple algebra $E_{\alpha}^{\mu}$ can be concisely written as

$$
E_{\alpha}^{\mu}=\frac{1}{\psi(1-\mathcal{L} H)} e_{\alpha}{ }^{\rho}\left(\partial \hat{H}^{-1}\right)_{\rho}^{\mu}
$$

where

$$
\begin{gathered}
\mathcal{L} H \equiv \mathcal{L}_{\mu}^{+} H^{--+\mu}, \quad \mathcal{L}_{\mu}^{+} \equiv e_{\mu}{ }^{\alpha} \mathcal{L}_{\alpha}^{+}, \quad e_{\mu}{ }^{\beta} e_{\alpha}{ }^{\mu}=\delta_{\alpha}^{\beta} \\
\hat{H}^{--+\rho} \equiv \frac{1}{1-\mathcal{L} H} H^{--+\rho} .
\end{gathered}
$$

It is a simple exercise to check that the constraint $(6.5)$ is identically satisfied by the expression (6.32).

Before going further, let us quote the transformation properties of the important quantities $e_{\alpha}^{\mu}, e_{\mu}{ }^{\alpha}, \mathcal{L}_{\mu}^{+}$introduced in subsec. 6.7 and 6.8

$$
\begin{aligned}
\delta e_{\alpha}{ }^{\mu} & =\lambda_{\alpha}{ }^{\beta} e_{\beta}{ }^{\mu}+2 \lambda e_{\alpha}{ }^{\mu}+e_{\alpha}{ }^{\rho} \hat{\partial}_{\rho}^{-} \lambda^{+\mu} \\
\delta e_{\nu}{ }^{\alpha} & =-e_{\nu}{ }^{\beta} \lambda_{\beta}{ }^{\alpha}-2 \lambda e_{\nu}{ }^{\alpha}-\hat{\partial}_{\nu}{ }^{-} \lambda^{+\mu} e_{\mu}{ }^{\alpha} \\
\delta \mathcal{L}_{\mu}^{+} & =-\hat{\partial}_{\mu}^{-} \lambda^{+\nu} \mathcal{L}_{\nu}^{+}-\hat{\partial}_{\mu}^{-} \lambda^{++}
\end{aligned}
$$

where

$$
\begin{gathered}
\hat{\partial}_{\mu}^{-} \equiv \partial_{\mu}^{-}+\mathcal{L}_{\mu}^{+} \partial^{--} \\
{\left[\hat{\partial}_{\mu}^{-}, \hat{\partial}_{\nu}^{-}\right]=2 \hat{\partial}_{\left[\mu^{-}\right.} \mathcal{L}_{\nu]}^{+} \partial^{--} \equiv 2 T_{\mu \nu} \partial^{--}} \\
\delta \hat{\partial}_{\mu}^{-}=-\hat{\partial}_{\mu}^{-} \lambda^{+\rho} \hat{\partial}_{\rho}^{-} \\
\delta T_{\mu \nu}=-\hat{\partial}_{\mu}^{-} \lambda^{+\rho} T_{\rho \nu}+\hat{\partial}_{\nu}^{-} \lambda^{+\rho} T_{\rho \mu}-T_{\mu \nu}\left(\partial^{--} \lambda^{++}+\mathcal{L}_{\rho}^{+} \partial^{--} \lambda^{+\rho}\right) .
\end{gathered}
$$

One immediately checks that $E_{\alpha}{ }^{\mu}$ given in (6.32) have the necessary transformation properties (6.1).

\section{$7 \lambda$-world geometry: quaternionic potentials}

In this Section we continue studying the quaternionic geometry in the $\lambda$-world representation. Here we at last reach the place where the quaternionic geometry potentials appear. 


\subsection{Expressing $\omega_{\alpha \beta}^{++}, \phi^{++}$and $H^{+3 \nu}$}

A new set of important relations arises from considering the commutator

$$
\left[\mathcal{D}^{++}, \mathcal{D}_{\alpha}^{-}\right]=\mathcal{D}_{\alpha}^{+}
$$

which is trivially satisfied in the $\tau$-world but leads to nontrivial restrictions while treating the $\lambda$-world geometry. The most informative relations follow from equating the coefficients of $\partial_{\mu}^{-}$and $\partial^{--}$in both sides of (7.1)

$$
\begin{gathered}
\mathcal{D}_{\lambda}^{++} e_{\alpha}{ }^{\mu}-\phi^{++} e_{\alpha}{ }^{\mu}-e_{\alpha}{ }^{\nu} \hat{\partial}_{\nu}{ }^{-} H^{+3 \mu}-\mathcal{L}_{\alpha}^{+} x_{A}^{+\mu}=0, \\
\mathcal{D}_{\lambda}^{++} \mathcal{L}_{\alpha}^{+}-\phi^{++} \mathcal{L}_{\alpha}^{+}-e_{\alpha}{ }^{\mu} \hat{\partial}_{\mu}{ }^{-} H^{+4}=0
\end{gathered}
$$

All the other equations associated with (7.1) can be shown to be satisfied in virtue of eqs. (7.2), (7.3) and those deduced previously.

Let us turn to extracting the consequences of eqs. (7.2), (7.3).

As a first corollary we note that the previously written constraint (6.9) is satisfied identically if one substitutes for $E_{\alpha}{ }^{\mu}$ its expression (6.32) and takes into account eqs. (7.2), (7.3) and (6.14). So we may forget about eq. (6.9). At this step we may also express the connections $\omega_{\alpha \gamma}^{++}$and $\phi^{++}$in terms of the analytic vielbeins

$$
\begin{aligned}
\omega_{\alpha \gamma}^{++} & =e_{(\alpha}{ }^{\mu} \Delta^{++} e_{\mu \gamma)}+e_{(\alpha}{ }^{\nu} \hat{\partial}_{\nu}{ }^{-} H^{+3 \mu} e_{\mu \gamma)}+\mathcal{L}_{(\alpha}^{+} x_{A}^{+\mu} e_{\mu \gamma)} \\
\phi^{++} & =\frac{1}{2 n}\left(e_{\mu}{ }^{\alpha} \Delta^{++} e_{\alpha}{ }^{\mu}-\hat{\partial}_{\nu}{ }^{-} H^{+3 \nu}-\mathcal{L}_{\mu}^{+} x_{A}^{+\mu}\right) .
\end{aligned}
$$

Further, let us rewrite (7.2), (7.3) as the equations for $e_{\mu}{ }^{\alpha}, \mathcal{L}_{\mu}^{+}$

$$
\begin{aligned}
& \mathcal{D}_{\lambda}{ }^{++} e_{\mu}{ }^{\alpha}+\phi^{++} e_{\mu}{ }^{\alpha}+e_{\nu}{ }^{\alpha} \hat{\partial}_{\mu}^{-} H^{+3 \nu}+e_{\nu}{ }^{\alpha} x_{A}^{+\nu} \mathcal{L}_{\mu}^{+}=0 \\
& \Delta^{++} \mathcal{L}_{\mu}^{+}+\mathcal{L}_{\nu}^{+} \hat{\partial}_{\mu}{ }^{-} H^{+3 \nu}+\mathcal{L}_{\mu}^{+}\left(\mathcal{L}_{\nu}^{+} x_{A}^{+\nu}\right)-\hat{\partial}_{\mu}{ }^{-} H^{+4}=0 .
\end{aligned}
$$

Then, introducing

$$
\begin{gathered}
\mathcal{L}^{+4} \equiv H^{+4}-\mathcal{L}_{\nu}^{+} H^{+3 \nu} \\
\delta \mathcal{L}^{+4}=-\partial^{++} \lambda^{++}-\mathcal{L}^{+4}\left(\partial^{--} \lambda^{++}\right)-\lambda^{++}\left(\mathcal{L}_{\nu}^{+} x_{A}^{+\nu}\right)-\mathcal{L}_{\nu}^{+} \partial^{++} \lambda^{+\nu}-\left(\mathcal{L}_{\nu}^{+} \partial^{--} \lambda^{+\nu}\right) \mathcal{L}^{+4},
\end{gathered}
$$

one may express the vielbein $H^{+3 \nu}$ from eq. (7.7) as a function of $\mathcal{L}^{+4}$ and $\mathcal{L}_{\mu}^{+}$

$$
H^{+3 \nu}=-\frac{1}{2} T^{\nu \mu}\left[\hat{\partial}_{\mu}^{-} \mathcal{L}^{+4}-\partial^{++} \mathcal{L}_{\mu}^{+}-\mathcal{L}^{+4} \partial^{--} \mathcal{L}_{\mu}^{+}-\mathcal{L}_{\mu}^{+}\left(\mathcal{L}_{\nu}^{+} x_{A}^{+\nu}\right)\right],
$$

where $T^{\nu \mu}$ is inverse to $T_{\mu \nu}$ defined in eq. 6.37), $T^{\nu \mu} T_{\mu \rho}=\delta_{\rho}^{\nu}$.

Thus we have succeeded in expressing the harmonic vielbein $H^{+3 \nu}$ and, in virtue of eqs. (6.16), the vielbeins entering into $\mathcal{D}_{\lambda}^{--}$, through two analytic and otherwise unconstrained objects, $\mathcal{L}_{\mu}^{+}\left(x_{A}^{+}, w^{ \pm i}\right)$ and $\mathcal{L}^{+4}\left(x_{A}^{+}, w^{ \pm i}\right)$. These objects, just as in the HK case, are fundamental objects of the quaternionic geometry, its potentials. As we see, the real advantage of considering the bi-harmonic extension of the quaternionic manifold is that the prepotentials naturally appear as the vielbeins associated with the partial derivative $\left(\partial^{--}-\partial / \partial z^{++}\right)$in the covariant derivatives $\mathcal{D}^{++}(5.9)$ and $\mathcal{D}_{\alpha}^{-}(6.18)$. 


\subsection{Fixing analytic $S p(1)$ weight gauge freedom}

Below we will demonstrate that all the geometric objects encountered earlier in this and previous Sections can be expressed through $\mathcal{L}_{\mu}^{+}\left(x_{A}^{+}, w^{ \pm i}\right)$ and $\mathcal{L}^{+4}\left(x_{A}^{+}, w^{ \pm i}\right)$ after fixing a gauge with respect to the analytic $S p(1)$ weight transformation (with the parameter $\left.\lambda\left(x_{A}^{+}, w^{ \pm i}\right)\right)$.

To see that this gauge freedom can be completely fixed, let us pass to $\tilde{e}_{\alpha}{ }^{\mu}$ and $\tilde{A}_{\alpha}^{\mu}$ (with $z_{A}^{0}$ factored out) and define

$$
\begin{gathered}
\tilde{e} \equiv \operatorname{det} \tilde{e}_{\alpha}{ }^{\mu}, \quad \tilde{A} \equiv \operatorname{det} \tilde{A}_{\alpha}{ }^{\mu}=\left(1-\mathcal{L}_{\mu}^{+} H^{--+\mu}\right) \tilde{e} \\
\delta \tilde{e}=\left(\hat{\partial}_{\mu}{ }^{-} \lambda^{+\mu}+2 n \lambda\right) \tilde{e}, \quad \delta \tilde{A}=\left(\partial_{\mu}^{-} \lambda^{+\mu}+H^{--+\rho} \partial_{\rho}^{-} \lambda^{++}+2 n \lambda\right) \tilde{A} .
\end{gathered}
$$

Next, one introduces

$$
B=\psi \tilde{A}, \quad \delta B=\left[2(n+1) \lambda+\partial_{\mu}^{-} \lambda^{+\mu}-\partial^{--} \lambda^{++}\right] B
$$

and checks that $B$ is analytic,

$$
\Delta_{\alpha}^{+} B=0
$$

Thus, we have two independent analytic quantities, $B$ and $\tilde{e}$, possessing nontrivial and different transformation laws under the analytic $S p(1)$ weight transformations. Hence one may completely fix the gauge with respect to these transformations by gauging away some combination of $B$ and $\tilde{e}$. There exists a two-parameter family of such gauges

$$
\begin{gathered}
B^{\gamma} e^{\alpha}=1 \Rightarrow \\
\lambda=\frac{\gamma \partial^{--} \lambda^{++}-\alpha \mathcal{L}_{\mu}^{+} \partial^{--} \lambda^{+\mu}-(\gamma+\alpha) \partial_{\mu}^{-} \lambda^{+\mu}}{2[\gamma(n+1)+\alpha n]}, \quad \gamma(n+1)+\alpha n \neq 0 .
\end{gathered}
$$

As we will see, the most convenient choice is

$$
\gamma=-\alpha \quad \Rightarrow B=\tilde{e}, \quad \lambda=\frac{1}{2}\left(\partial^{--} \lambda^{++}+\mathcal{L}_{\mu}^{+} \partial^{--} \lambda^{+\mu}\right)
$$

Expressing $\psi$ from eqs. (7.11), (7.13)

$$
\psi=\frac{B}{\tilde{e}} \frac{1}{1-\mathcal{L} H},
$$

one finds that in the gauge (7.17) $\psi$ is a function entirely of the prepotentials $\mathcal{L}_{\mu}^{+}$and $\mathcal{L}^{+4}$

$$
\psi=\frac{1}{1-\mathcal{L} H}
$$

Below we will see that even before fixing the gauge one of the quantities $B$ and $\tilde{e}$ can be expressed through the quaternionic potentials. 


\subsection{Expressing $\rho_{\mu}^{-}$and $e_{\mu}{ }^{\alpha}$}

In this subsection we will express the vielbeins $\rho_{\mu}^{-}$and $e_{\mu}{ }^{\alpha}$ (defined by eqs. (5.29) and (6.20) ) in terms of the prepotentials.

To this end we need to consider the commutator

$$
\left[\mathcal{D}^{--}, \mathcal{D}_{\alpha}^{-}\right]=0
$$

whose validity is evident while it is considered in the $\tau$-world. The relations relevant to our purpose follow from vanishing of the torsion components $T_{\alpha}^{-3(++)}$ and $T_{\alpha}^{-3(+\mu)}$ :

$$
\begin{aligned}
T_{\alpha}^{-3(++)}=0 \Rightarrow & \mathcal{D}_{\lambda}^{--} \mathcal{L}_{\alpha}^{+}+E_{\alpha}^{-2 \mu} \partial_{\mu}^{+} \ln \psi-e_{\alpha}{ }^{\rho} \hat{\partial}_{\rho}^{-} \ln \psi+2 \rho_{\alpha}^{-}=0 \\
T_{\alpha}^{-3(+\mu)}=0 \Rightarrow & \mathcal{D}_{\lambda}{ }^{--} e_{\alpha}{ }^{\mu}-\mathcal{D}_{\lambda}^{--} \mathcal{L}_{\alpha}^{+} H^{--+\mu}+E_{\alpha}^{-2 \rho} \partial_{\rho}^{+} H^{--+\mu} \\
& -e_{\alpha}{ }^{\rho} \hat{\partial}_{\rho}^{-} H^{--+\mu}=0
\end{aligned}
$$

where $E_{\alpha}^{-2 \rho}$ is given by (6.19). All the remaining relations are satisfied either as cosequences of these essential ones or in virtue of those obtained earlier.

Expressing $E_{\alpha}^{-2 \rho}$ from eq. (7.22) and substituting the result into eq. (7.21) one gets the expression for $\rho_{\mu}^{-} \equiv e_{\mu}^{\alpha} \rho_{\alpha}^{-}=\tilde{e}_{\mu}^{\alpha} \tilde{\rho}_{\alpha}^{-}$:

$$
\rho_{\mu}^{-}=-\frac{1}{2}\left(\hat{\partial}_{\mu}^{-} \ln \frac{\tilde{e}}{B}+\partial^{--} \mathcal{L}_{\mu}^{+}+2 \hat{H}^{--+\nu} T_{\nu \mu}\right) .
$$

It is a simple exercise to check that $\rho_{\mu}^{-}$possesses the needed transformation properties

$$
\delta \rho_{\mu}^{-}=\hat{\partial}_{\mu}^{-} \lambda-\hat{\partial}_{\mu}^{-} \lambda^{+\nu} \rho_{\nu}^{-}
$$

We leave it to the reader to prove the validity of the relation (6.12). One must take into account the relation (5.24) whence

$$
\Delta_{\alpha}^{+} \phi=-\Delta_{\alpha}^{+}\left[\psi\left(1-\Delta^{--} \phi^{++}\right)\right]=e_{\alpha}{ }^{\mu} \hat{\partial}_{\mu}^{-} \phi^{++}-\mathcal{L}_{\alpha}^{+},
$$

use one more reprsentation for the harmonic $S p(1)$ connection $\phi^{++}$following from eq.(6.14)

$$
\phi^{++}=\frac{1}{2}\left(\mathcal{L}_{\nu}^{+} \partial^{--} H^{+3 \nu}+\mathcal{L}_{\mu}^{+} x_{\nu}^{+\mu}-\partial^{--} H^{+4}-\Delta^{++} \ln \frac{\tilde{e}}{B}\right)
$$

and, finally, enforce the gauge (7.17).

It remains to find the appropriate expression for $e_{\mu}{ }^{\alpha} \equiv\left(z_{A}^{0}\right)^{-2} \tilde{e}_{\mu}{ }^{\alpha}$. Like in the HK case, it is more convenient to deal with the "metric" $h_{\mu \nu} \equiv\left(z_{A}^{0}\right)^{-2} \tilde{h}_{\mu \nu}$

$$
h_{\mu \nu} \equiv e_{\mu}^{\alpha} e_{\nu \alpha}=-h_{\nu \mu}, \quad \tilde{h}_{\mu \nu} \equiv \tilde{e}_{\mu}^{\alpha} \tilde{e}_{\nu \alpha}, \quad \delta \tilde{h}_{\mu \nu}=-\hat{\partial}_{\mu}^{-} \lambda^{+\rho} \tilde{h}_{\rho \mu}-2 \lambda \tilde{h}_{\mu \nu} .
$$

Being rewritten for $\tilde{h}_{\mu \nu}$, eq. (7.2) takes the form

$$
\Delta^{++} \tilde{h}_{\rho \mu}+2 \phi^{++} \tilde{h}_{\rho \mu}-2 \hat{\partial}_{[\rho}^{-} H^{+3 \sigma} \tilde{h}_{\mu] \sigma}-2 \mathcal{L}_{[\rho}^{+} x_{A}^{+\nu} \tilde{h}_{\mu] \nu}=0
$$

Let us now apply to the constraints (6.6), (6.26) for the vielbein $\rho_{\mu}^{-}$. Put together, they can be rewritten as

$$
e_{\mu}^{\alpha} \Delta_{\alpha}^{+} \rho_{\nu}^{-}=\tilde{e}_{\mu}{ }^{\alpha} \tilde{\Delta}_{\alpha}^{+} \rho_{\nu}^{-}=-\tilde{h}_{\mu \nu} R
$$


Substituting (7.23) into (7.28) and keeping in mind the analyticity of $\tilde{e} / B, \mathcal{L}_{\mu}^{+}$and $T_{\mu \nu}$, one gets, after some algebra

$$
\tilde{h}_{\mu \nu}=R^{-1}\left(\frac{\tilde{e}}{B}\right) T_{\mu \nu},
$$

whence

$$
\tilde{e}=(\operatorname{det} T)^{-\frac{1}{2(n+1)}} B^{\frac{n}{n+1}}(R)^{\frac{n}{n+1}} .
$$

Thus, we have expressed $\tilde{h}_{\mu \nu}$ (and, hence, $\tilde{e}_{\mu}^{\alpha}$, up to an analytic frame $S p(n)$ rotation) in terms of the prepotential $\mathcal{L}_{\mu}^{+}$and the analytic "compensator" $B$. In the gauge (7.17)

$$
\begin{gathered}
\tilde{h}_{\mu \nu}=R^{-1} T_{\mu \nu}, \\
\tilde{e}=(\operatorname{det} T)^{-\frac{1}{2}} R^{n} .
\end{gathered}
$$

It remains to check the identity (7.27). Using the explicit form (6.37) of $T_{\mu \nu}$, it is easy to obtain

$$
\begin{aligned}
& \Delta^{++} T_{\mu \nu}-2 \hat{\partial}_{[\mu}^{-} H^{+3 \rho} T_{\nu] \rho}-2 \mathcal{L}_{[\mu}^{+} x^{+\rho} T_{\nu] \rho} \\
& +T_{\mu \nu}\left(\mathcal{L}_{\rho}^{+} x^{+\rho}-\partial^{--} H^{+4}+\mathcal{L}_{\rho}^{+} \partial^{--} H^{+3 \rho}\right)=0 .
\end{aligned}
$$

Remembering (7.25), one observes that the expression within the parentheses is just $2 \phi^{++}$ in the gauge (7.17). This proves (7.27).

\subsection{Full structure of $\mathcal{D}^{--}$and $\mathcal{D}_{\alpha}^{-}$}

To finish expressing the objects of the quaternionic geometry through analytic potentials, we have to completely specify the structure of the derivatives $\mathcal{D}^{--}$and $\mathcal{D}_{\alpha}^{-}$by finding appropriate expressions for the $S p(n)$ connection $\omega^{--}$and the vielbein $E_{\alpha}^{-2 \mu}$ defined in eq. (6.19). To this end, we need to express $\mathcal{D}_{\lambda}^{--} E_{\alpha}^{\mu}$. Comparing the representation for $\mathcal{D}_{\lambda}^{--} E_{\alpha}^{\mu}$ following from eq. (7.22) with the result of the explicit action of $\mathcal{D}_{\lambda}^{--}$on the expression (6.29), one finds

$$
\begin{aligned}
\left(\mathcal{D}_{\lambda}^{--} E_{\alpha}^{\mu}\right) E_{\mu \beta}= & -\frac{1}{2}\left[\Omega_{\beta \alpha} \Delta^{--} \ln \psi-A_{\alpha}{ }^{\sigma}\left(\partial_{\sigma}^{-} H^{--+\rho}\right) A_{\rho \beta}\right. \\
& -A_{\alpha}{ }^{\mu}\left(\partial H^{-1}\right)_{\mu}{ }^{\sigma} \partial_{\sigma}^{+} H^{-3 \nu} \partial_{\nu}^{-} H^{--+\rho} A_{\rho \beta} \\
& \left.+A_{\alpha}{ }^{\mu}\left(\partial H^{-1}\right)_{\mu}{ }^{\sigma} \Delta^{--} \partial_{\sigma}^{+} H^{--+\rho} A_{\rho \beta}\right] .
\end{aligned}
$$

Further, let us apply the derivative $\mathcal{D}^{++}=\mathcal{D}_{\lambda}^{++}+\phi^{++} Z^{0}$ (we are not interested in terms with the $z^{ \pm \pm}$derivatives) to $\left(z_{A}^{0}\right)^{-2} \psi\left(\mathcal{D}_{\lambda}^{--} E E^{-1}\right)_{\alpha \beta}$. Using the commutation relation (6.17) and the constraint (6.14), one gets

$$
\mathcal{D}^{++}\left[\left(z_{A}^{0}\right)^{-2} \psi\left(\mathcal{D}_{\lambda}^{--} E E^{-1}\right)_{\alpha \beta}\right]=\Omega_{\alpha \beta}\left(z_{A}^{0}\right)^{-2} \psi \Delta^{--} \phi^{++},
$$

whence it immediately follows that

$$
\begin{gathered}
\mathcal{D}_{\lambda}^{--} E_{(\alpha}^{\mu} E_{\mu \beta)}=0 \\
\mathcal{D}_{\lambda}^{--} E_{[\alpha}^{\mu} E_{\mu \beta]} \sim \Omega_{\alpha \beta} .
\end{gathered}
$$


From eq. (7.36) we find $\omega_{\alpha \beta}^{--}$as a function of $E_{\alpha}{ }^{\mu}$ and, hence, of the prepotentials $\mathcal{L}^{+4}$ and $\mathcal{L}_{\alpha}^{+}$

$$
\omega_{\alpha \beta}^{--}=E_{(\alpha}{ }^{\mu} \Delta^{--} E_{\mu \beta)}^{-1},
$$

while eqs. (7.37) and (7.34) put together yield

$$
\begin{gathered}
\mathcal{D}_{\lambda}^{--} E_{\alpha}^{\mu}=E_{\alpha}^{\mu} N^{--} \\
N^{--} \equiv \frac{1}{4 n}\left[\partial_{\mu}^{-} H^{--+}+\partial_{\mu}^{+} H^{-3 \mu}-\Delta^{--} \ln (\operatorname{det} \partial H)-2 n \Delta^{--} \ln \psi\right] .
\end{gathered}
$$

Thus, $\mathcal{D}_{\lambda}^{--} E_{\alpha}^{\mu}$, the last $\lambda$-world geometric object to be specified, also turned out expressed in terms of the quaternionic potentials.

To be convinced that eq. (7.39) is correct, one may compare the transformation properties of its both sides. It is not difficult to find

$$
\begin{aligned}
\delta \mathcal{D}_{\lambda}^{--} E_{\alpha}^{\mu}= & \lambda_{\alpha}{ }^{\beta} \mathcal{D}_{\lambda}^{--} E_{\beta}^{\mu}+\left(\mathcal{D}_{\lambda}^{--} E_{\alpha}^{\rho}\right) \partial_{\rho}^{+} \lambda^{-\mu}-\Delta^{--} \lambda E_{\alpha}^{\mu} \\
& +E_{\alpha}^{\mu} \Delta^{--}\left[\frac{1}{2 n}\left(\partial_{\rho}^{+} \lambda^{-\rho}\right)-\lambda\right]+\left(\Delta^{--} \lambda^{++}\right) \mathcal{D}_{\lambda}^{--} E_{\alpha}^{\mu}
\end{aligned}
$$

where we have used the property

$$
\begin{aligned}
\mathcal{D}^{++}\left[\left(z_{A}^{0}\right)^{-2} \psi \Delta^{--} \partial_{\nu}^{+} \lambda^{-\mu}\right] & =-\left(z_{A}^{0}\right)^{-2} \psi\left(\Delta^{--} \lambda^{++}\right) \delta_{\nu}^{\mu} \\
\Rightarrow \Delta^{--} \partial_{\nu}^{+} \lambda^{-\mu} & =\frac{1}{2 n} \Delta^{--}\left(\partial_{\rho}^{+} \lambda^{-\rho}\right) \delta_{\nu}^{\mu}
\end{aligned}
$$

(recall that in the HK case [2] the r.h.s. of the analogous identity is zero). Directly evaluating the transformation properties of different pieces in $\mathrm{N}^{--}$, one finds

$$
\delta N^{--}=\left(\Delta^{--} \lambda^{++}\right) N^{--}+\frac{1}{2 n} \Delta^{--}\left(\partial_{\rho}^{+} \lambda^{-\rho}\right)-\Delta^{--} \lambda
$$

that suggests the same transformation law for both sides of (7.39).

\subsection{The quaternionic metric in the $\lambda$-basis}

Summarizing the computations of the previous subsections, one may write the covariant derivatives of the quaternionic geometry (their parts involving no derivatives with respect to the coordinates $z^{ \pm \pm}$) in the following form

$$
\begin{aligned}
\mathcal{D}_{\alpha}^{+}= & \frac{1}{\psi(1-\mathcal{L} H)} e_{\alpha}{ }^{\rho}\left(\partial \hat{H}^{-1}\right)_{\rho}{ }^{\mu} \partial_{\mu}^{+} \equiv E_{\alpha}^{+\mu-} \partial_{\mu}^{+} \\
\mathcal{D}_{\alpha}^{-}= & \left(z_{A}^{0}\right)^{-2}\left\{\left[\psi E_{\alpha}{ }^{\mu} N^{-2}+\mathcal{L}_{\alpha}^{+} H^{-3 \mu}-\psi E_{\alpha}{ }^{\rho} \partial_{\rho}^{+} H^{-3 \mu}+z_{A}^{--} E_{\alpha}{ }^{\mu}\right] \partial_{\mu}^{+}\right. \\
& \left.-e_{\alpha}{ }^{\mu} \hat{\partial}_{\mu}^{-}-\rho^{-}\left(z_{A}^{0} \frac{\partial}{\partial z_{A}^{0}}\right)-\Delta_{\alpha}^{+}\left(\psi \omega^{--}\right)\right\} \\
\equiv & E_{\alpha}{ }^{-\mu-} \partial_{\mu}^{+}+E_{\alpha}{ }^{-\mu+} \hat{\partial}_{\mu}^{-}+\omega_{\alpha}^{-} Z^{0}-\omega_{\alpha(\sigma \rho)}^{-} \Gamma^{(\sigma \rho)} .
\end{aligned}
$$

Defining

$$
g_{(\lambda)}^{M N}=-E^{+M \alpha} E_{\alpha}^{-N}+E^{-M \alpha} E_{\alpha}^{+N}, \quad M, N=(\nu+, \nu-),
$$


one obtains

$$
\begin{gathered}
g_{(\lambda)}^{\mu+\nu+}=0 \\
g_{(\lambda)}^{\mu+\nu-}=g_{(\lambda)}^{\nu-\mu+}=E^{-\mu+\alpha} E_{\alpha}^{+\nu-}=R T^{\mu \rho}\left(\partial \hat{H}^{-1}\right)_{\rho}{ }^{\nu} \\
g_{(\lambda)}^{\mu-\nu-}=-E^{+\mu-\alpha} E_{\alpha}^{-\nu-}+E^{-\mu-\alpha} E_{\alpha}^{+\nu-} \\
=-2 R T^{\rho \lambda}\left(\partial \hat{H}^{-1}\right)_{\lambda}{ }^{\sigma}\left(\partial \hat{H}^{-1}\right)_{\rho}{ }^{(\mu} \partial_{\sigma}^{+} \hat{H}^{-3 \nu)} .
\end{gathered}
$$

We stress that the dependence on $z_{A}^{--}, z_{A}^{0}$ present in $(7.43),(7.45)$ completely drops out from the $\lambda$-basis metric which is given on the manifold $\left\{x_{A}^{+}, x_{A}^{-}, w^{ \pm i}\right\}$. Of course, one may easily check that the metric possesses, as in the HK case, the property of the covariant independence of harmonics. It is also to the point here to mention that the bridge from the $S p(n)-\tau$-frame to the $\lambda$-frame is expressed in terms of the harmonic connection $\omega^{++}$by the same relation as in the HK case, so we do not give it here.

\subsection{Gauges}

Similarly to the HK case, in practice it is advantageous to fix, in one or another way, the gauges with respect to different $\lambda$-transformation, thereby decreasing the number of independent quantities originally present in the theory. Some gauges (eqs. (5.21), (5.22), (7.15), (7.17)) have been already imposed in the process of solving quaternionic geometry constraints. Here we enforce further gauge-fixing analogous to the one employed in the HK case [2].

First, one may put $\left([R]=\mathrm{cm}^{-2}\right)$

$$
\begin{gathered}
\mathcal{L}_{\mu}^{+}=R \Omega_{\mu \nu} x_{A}^{+\nu} \equiv R x_{A \mu}^{+} \Rightarrow T_{\mu \nu}=-R \Omega_{\mu \nu} \\
\lambda_{\mu}^{+}+\hat{\partial}_{\mu}^{-} \lambda^{+\nu} x_{A \nu}^{+}+R^{-1} \hat{\partial}_{\mu}^{-} \lambda^{++}=0 \Rightarrow \lambda_{\mu}^{+}=-\frac{1}{2} R^{-1} \hat{\partial}_{\mu}^{-} \tilde{\lambda}^{++}, \tilde{\lambda}^{++} \equiv \lambda^{++}+R \lambda^{+\nu} x_{A \nu}^{+}
\end{gathered}
$$

Note the relation

$$
\hat{\partial}_{[\rho}^{-} \lambda_{\mu]}^{+}=-\frac{1}{2} R^{-1} T_{\rho \mu} \partial^{--} \tilde{\lambda}^{++}=\frac{1}{2} \Omega_{\rho \mu} \partial^{--} \tilde{\lambda}^{++} .
$$

In this gauge the transformation law of the remaining (non-removable) prepotential $\mathcal{L}^{+4}$ is greatly simplified

$$
\delta \mathcal{L}^{+4}=-\partial^{++} \tilde{\lambda}^{++}-\left(\partial^{--} \tilde{\lambda}^{++}\right) \mathcal{L}^{+4}
$$

or, in the active form

$$
\begin{aligned}
\delta^{*} \mathcal{L}^{+4}\left(x_{A}^{+}, w^{ \pm i}\right) \simeq & \mathcal{L}^{+4^{\prime}}\left(x_{A}^{+}, w^{ \pm i}\right)-\mathcal{L}^{+4}\left(x_{A}^{+}, w^{ \pm i}\right)=-\partial^{++} \tilde{\lambda}^{++}-\left(\partial^{--} \tilde{\lambda}^{++}\right) \mathcal{L}^{+4} \\
& +\tilde{\lambda}^{++} \partial^{--} \mathcal{L}^{+4}+\frac{1}{2} R^{-1} \hat{\partial}^{-\mu} \tilde{\lambda}^{++} \hat{\partial}_{\mu}^{-} \mathcal{L}^{+4}
\end{aligned}
$$

Note that the isometries of quaternionic metric correspond to

$$
\delta^{*} \mathcal{L}^{+4}=0
$$


Denoting the relevant transformation parameter in $(7.54)$ by $K^{++}$,

$$
K^{++} \equiv K_{A}^{++} c_{A}
$$

with $c_{A}$ being constant infinitesimal parametries of the isometries, one obtains from (7.54) the equation for $K^{++}$

$$
\partial^{++} K^{++}+\left(\partial^{--} K^{++}\right) \mathcal{L}^{+4}-K^{++} \partial^{--} \mathcal{L}^{+4}-\frac{1}{2} R^{-1} \hat{\partial}^{-\mu} K^{++} \hat{\partial}_{\mu}^{-} \mathcal{L}^{+4}=0
$$

(and the same for $K_{A}^{++}$). The quantity $K_{A}^{++}$encodes all the information about isometries of the quaternionic manifold with the given potential $\mathcal{L}^{+4}$ and can naturally be called quaternionic Killing potential [10].

Transformations (7.53), (7.54) and the equation for Killing potential (7.56) have been deduced earlier in [10] starting with the harmonic superspace action of $N=2$ matter in $N=2 \mathrm{SG}$ background. Here we rediscovered the same objects and relations on the pure geometrical ground, starting from the unconstrained prepotential formulation of quaternionic geometry. One-to-one correspondence between this formulation of quaternionic geometry and the harmonic superspace description of $N=2$ matter coupled to $N=2$ SG will be discussed in Sect. 10.

One more gauge also having a prototype in the HK case allows one to "solder" the $\lambda$-frame $S p(n)$ transformations with the diffeomorphisms of the analytic subspace

$$
\begin{gathered}
\tilde{e}_{\alpha}{ }^{\mu}=\delta_{\alpha}^{\mu} \Rightarrow \lambda_{\alpha \beta}+\lambda \Omega_{\alpha \beta}+\hat{\partial}_{\alpha}^{-} \lambda_{\beta}^{+}=0 \\
\Rightarrow \quad \lambda_{\alpha \beta}=-\hat{\partial}_{(\alpha}^{-} \lambda_{\beta}^{+} \quad \lambda=\frac{1}{2}\left(\partial^{--} \tilde{\lambda}^{++}\right) .
\end{gathered}
$$

One may see that (7.58) completely agrees with the previously imposed gauges (7.15) $\tilde{B}=\tilde{e}$ and $\tilde{B}=$ const or $\tilde{e}=$ const. These gauges now coincide in view of (7.57).

\section{Example: homogeneous manifold $S p(n+1) / S p(1) \times$ $S p(n)$}

Here we will consider the simplest example of $4 n$ dimensional quaternionic manifold corresponding to the choice $\mathcal{L}^{+4}=0$. As opposed to the HK case where such an option results in a trivial flat manifold, in the case under consideration we are left with a curved homogeneous space $S p(n+1) / S p(1) \times S p(n)($ or $S p(n, 1) / S p(1) \times S p(n)$, for the negative value of the parameter $R$ ) which can thus be regarded as the "maximally flat" connected $4 n$ dimensional quaternionic manifold.

The choice

$$
\mathcal{L}^{+4}=0
$$

in the Killing potential equation (7.56) reduces the latter to the following one (we assume that all the gauges employed above are imposed)

$$
\partial^{++} K^{++}\left(x^{+}, w\right)=0
$$


the general solution of which is

$$
\begin{aligned}
K^{++} & =w^{+i} w^{+j} c_{(i j)}+x^{\mu+} x^{\nu+} c_{(\mu \nu)}+x^{\mu+} w^{+i} c_{\mu i} \\
\lambda_{\mu}^{+} & \left.=-\frac{1}{2} R^{-1}\left(\partial_{\mu}^{-}+R x_{\mu}^{+} \partial^{--}\right) K^{++}\right) \\
& =-\frac{1}{2} R^{-1}\left[w^{+i} c_{\mu i}+2 x^{+\nu} c_{(\mu \nu)}+R x_{\mu}^{+}\left(2 w^{+i} w^{-j} c_{(i j)}+x^{\mu+} w^{-i} c_{\mu i}\right)\right],
\end{aligned}
$$

whence

$$
\lambda^{++}=K^{++}-R \lambda^{+\nu} x_{\nu}^{+}+w^{+i} w^{+j} c_{(i j)}+\frac{1}{2} x^{\mu+} w^{+i} c_{\mu i} .
$$

Studying the Lie algebra of the variations

$$
\delta x^{\mu+}=\lambda^{+\mu}, \quad \delta w^{-i}=0, \quad \delta w^{+i}=\lambda^{++} w^{-i},
$$

one finds that it coincides with that of $S p(n+1)$ (or with $S p(n, 1)$ for $R<0$ in (8.4)), the parameters $c_{(i j)}, c_{(\mu \nu)}, c_{\mu i}$ being associated, respectively, with the generators of the subgroups $S p(1), S p(n)$ and with those of the coset $S p(n+1) / S p(1) \times S p(n)$. It is easy to show that in this case

$$
\begin{gathered}
H^{+3 \nu}=0, H^{+4}=0, H^{--+\mu}=x^{-\mu}, H^{-3 \mu}=0 \\
\Delta^{ \pm \pm}=\partial^{ \pm \pm}+x^{ \pm \rho} \partial_{\rho}^{ \pm}, 1-\mathcal{L} H=1+R x^{+\mu} x_{\mu}^{-} \equiv 1+\frac{1}{2} R x^{2} \\
\rho_{\mu}^{-}=\frac{1}{1+\frac{1}{2} R x^{2}} x_{\mu}^{-}, \quad E_{\alpha}{ }^{\mu}=\left(1+\frac{1}{2} R x^{2}\right)\left(\delta_{\alpha}^{\mu}-R x_{\alpha}^{+} x^{-\mu}\right) \\
E_{\mu \beta}=\frac{1}{1+\frac{1}{2} R x^{2}}\left(\Omega_{\beta \mu}+R \frac{x_{\mu}^{+} x_{\beta}^{-}}{1+\frac{1}{2} R x^{2}}\right) \\
\omega_{\alpha \beta}^{++}=R x_{\alpha}^{+} x_{\beta}^{+}, \quad \omega_{\alpha \beta}^{--}=\frac{R}{1+\frac{1}{2} R x^{2}} x_{\alpha}^{-} x_{\beta}^{-} .
\end{gathered}
$$

It is also easy to check the covariant independence of $E_{\alpha}{ }^{\mu}$ of the harmonics

$$
\begin{aligned}
\mathcal{D}^{--} E_{\alpha}{ }^{\mu} & =\left(x^{-\rho} \partial_{\rho}^{-}\right) E_{\alpha}{ }^{\mu}+\left(\omega^{--}\right)_{\alpha}{ }^{\beta} E_{\beta}{ }^{\mu} \\
& =\left(1+\frac{1}{2} R x^{2}\right) x_{\alpha}^{-} x^{-\mu}-x_{\alpha}^{-} x^{-\mu}-\frac{1}{2} R x_{\alpha}^{-} x^{-\mu}\left(x^{2}\right)=0,
\end{aligned}
$$

as well as to verify all the remaining general relations derived earlier, to find the induced group parameters $\lambda, \lambda^{-\mu}$, etc. The nonvanishing component of the $\lambda$-world metric is given by the following simple expression

$$
g^{\mu+\nu-}=-\left(1+\frac{R}{2} x^{2}\right)\left(\Omega^{\mu \nu}-R x^{+\mu} x^{-\nu}\right) .
$$

The bridges can be shown to vanish in the present case, so $w_{A}^{ \pm i}=w^{ \pm i}$ and $\lambda^{++}=\tau^{++}$, and also

$$
x^{\mu i}=-x^{\mu+} w^{-i}+x^{\mu-} w^{+i} .
$$


To find the $\tau$-world metric, one should convert the indices $\mu, \nu$ in (8.12) with the matrices

$$
\hat{\partial}_{\mu}^{-} x^{\rho k}=-\delta_{\mu}^{\rho} w^{-k}+R x_{\mu}^{+} x^{-\rho} w^{-k}, \quad \partial_{\nu}^{+} x^{\sigma i}=\delta_{\nu}^{\sigma} w^{+i}
$$

and symmetrize indices $\rho k, \sigma i$ (without factor $1 / 2$ ). One gets

$$
\begin{aligned}
& g^{\rho k, \sigma i}=\epsilon^{k i}\left(1+\frac{R}{2} x^{2}\right)\left(\Omega^{\rho \sigma}-R x^{\rho j} x_{j}^{\sigma}\right), \\
& g_{\sigma i, \rho k}=\frac{\epsilon_{i k}}{1+\frac{R}{2} x^{2}}\left(\Omega_{\sigma \rho}-R \frac{x_{\sigma}^{j} x_{\rho j}}{1+\frac{R}{2} x^{2}}\right)
\end{aligned}
$$

that coincides with the metric of the space $S p(n+1) / S p(1) \times S p(n)$ in the appropriate projective coordinates (we work with the standard dimension 1 coordinates).

Finally, we leave it to the reader to reproduce all the formulas given here starting with the standard nonlinear realization description of the coset space $S p(n+1) / S p(1) \times S p(n)$, defining the corresponding Cartan forms, etc. In order to do this unambigously, one needs to extend this space to the harmonic one by introducing the harmonics $u^{ \pm i}$ on the extra automorphism $S U(2)$ (acting on the $S p(1)$ indices of the $S p(n+1)$ generators) and then to identify the analytic subspace $\left\{x_{\mu}^{+}, w^{ \pm i}\right\}$ with the following coset of $S p(n+1) \times S U(2)_{A}$

$$
\left\{x_{\mu}^{+}, w^{ \pm i}\right\}=\frac{S p(n+1) \times S U(2)_{A}}{\left(L^{(\mu \nu)}, P^{\mu+}, I^{++}, I^{0}, I^{--}-T^{--}, T^{0}\right)},
$$

where $I$ and $T$ denote the generators of the subgroups $S p(1) \subset S p(n+1)$ and $S U(2)_{A}$, respectively, $L^{(\mu \nu)}$ stands for the generators of $S p(n) \subset S p(n+1)$ and $P^{\mu+}$ for those of the coset $S p(n+1) / S p(1) \times S p(n)$ generators $P^{\mu i}$ which commute with $I^{++}, I^{--}-T^{--}$ (their number equals $2 n$ ).

In Sect. 11 we will discuss less trivial examples of homogeneous quaternionic manifolds with $\mathcal{L}^{+4} \neq 0$.

\section{HK manifolds as a contraction of the quaternionic ones}

Here we show that in the limit $R \rightarrow 0$ the formulation of quaternionic geometry given in the previous Sections goes over to that of the HK one with the central charge operators included [2].

Let us begin with the $\tau$-world constraints and consider first what happens in this limit with the bi-harmonic space $\left\{x^{\mu i}, w^{ \pm i}, z^{++}, z^{--}, z^{0}\right\}$. Rescaling $z$-coordinates as

$$
z^{ \pm \pm} \equiv R \tilde{z}^{ \pm \pm}, \quad z^{0} \equiv \exp \left\{R \tilde{z}^{0}\right\}
$$

and defining

$$
\tilde{Z}^{ \pm \pm} \equiv R Z^{ \pm \pm}, \quad \tilde{Z}^{0} \equiv R \tilde{Z}^{0}
$$

one finds that in the limit $R \rightarrow 0$ the expressions (3.16), (3.17) become

$$
\tilde{Z}^{0}=\frac{\partial}{\partial \tilde{z}^{0}}, \quad \tilde{Z}^{ \pm \pm}=\frac{\partial}{\partial \tilde{z}^{\mp \mp}}, \quad w^{ \pm i}=u^{ \pm i}
$$




$$
\begin{gathered}
{\left[\tilde{Z}^{0}, \tilde{Z}^{ \pm \pm}\right]=0, \quad\left[\tilde{Z}^{++}, \tilde{Z}^{--}\right]=0} \\
\mathcal{D}^{++}=\partial_{u}^{++}+\tilde{z}^{++} \frac{\partial}{\partial \tilde{z}^{0}}+2 \tilde{z}^{0} \frac{\partial}{\partial \tilde{z}^{--}} \\
\mathcal{D}^{--}=\partial_{u}^{--}+2 \tilde{z}^{0} \frac{\partial}{\partial \tilde{z}^{++}}+\tilde{z}^{--} \frac{\partial}{\partial \tilde{z}^{0}} \\
\mathcal{D}^{0}=\partial_{u}^{0}+2\left(\tilde{z}^{++} \frac{\partial}{\partial \tilde{z}^{++}}-\tilde{z}^{--} \frac{\partial}{\partial \tilde{z}^{--}}\right) .
\end{gathered}
$$

Thus, $\tilde{Z}^{++}, \tilde{Z}^{--}, \tilde{Z}^{0}$ become just the central charge operators introduced in [2] as a useful device for solving the HK geometry constraints in a regular way. The bi-harmonic space goes over to the standard twistor-harmonic extension of the $\tau$-basis HK manifold enlarged by the extra central charge coordinates

$$
\left\{x^{\mu i}, w^{ \pm j}, z\right\} \Rightarrow\left\{x^{\mu i}, u^{ \pm j}, \tilde{z}\right\} .
$$

Further, the quaternionic geometry defining constraint (4.10) formally preserves its form in the limit $R \rightarrow 0$

$$
\left[\mathcal{D}_{\alpha}^{+}, \mathcal{D}_{\beta}^{+}\right]_{\rho}^{\rho^{\prime}}=-2 \delta_{\rho}^{\rho^{\prime}} \Omega_{\alpha \beta} \tilde{Z}^{++}, \quad \mathcal{D}_{\alpha}^{+}=u^{+i} \mathcal{D}_{\alpha i}, \quad \tilde{Z}^{++}=-u^{+i} u^{+j} Z_{(i j)} .
$$

However, because the holonomy $S p(1)$ contracts into the trivial algebra of three flat $\tilde{z}$ translations (9.4), the status of (9.7) radically changes. Namely, $\mathcal{D}_{\alpha}^{+}$has now the following structure (cf. (4.23))

$$
\mathcal{D}_{\alpha}^{+}=\hat{E}_{\alpha}^{+\mu k} \partial_{\mu k}-\hat{\omega}_{\alpha(\rho \sigma)}^{+} \Gamma^{\rho \sigma}-\hat{\omega}_{\alpha}^{+-+} \frac{\partial}{\partial \tilde{z}^{0}}-\hat{\omega}_{\alpha}^{+--} \frac{\partial}{\partial \tilde{z}^{--}}-\hat{\omega}_{\alpha}^{+3} \frac{\partial}{\partial \tilde{z}^{++}},
$$

where hat means that we consider the $R=0$ contraction of the corresponding objects of the quaternionic geometry. Note that (9.8) contains no $\tilde{z}^{0}$ dependence because $z^{0}=$ $\exp \left\{R \tilde{z}^{0}\right\} \rightarrow 1$ as $R \rightarrow 0$. It is easy to see that (9.7) produces for $\hat{E}_{\alpha}^{+\mu k}, \hat{\omega}_{\alpha(\rho \sigma)}^{+}$the standard HK constraints while in the quaternionic case the corresponding constraints essentially involved the vielbeins multiplying the $z$-derivatives. Thus, in the $\tau$-world eq. (9.7) (accompanied by the the evident harmonic commutation relations) amounts to the familiar constraints of HK geometry.

When passing to the $\lambda$-world, we have to rescale $z_{A}^{0}, z_{A}^{ \pm}$in the same fashion as in eq. (9.1) and simultaneously to rescale the bridges and the gauge group parameters. There is a minor difference of the resulting $\lambda$-world HK constraints as compared with the corresponding ones obtained in [2]. The difference stems from the fact that in the quaternionic case we are led to include $z_{A}^{0}$ into the analytic subspace (together with $z_{A}^{++}$) in order to have analytic $S p(1)$ weight transformations. No any $S p(1)$ weight structures survive in the $R=0$ contraction limit, so one can, in principle, restrict oneself to considering the analytic space involving only $\tilde{z}_{A}^{++}$(treating $\tilde{z}_{A}^{0}$ on equal footing with $\tilde{z}_{A}^{--}$) and this is just what has been done in [2]. Contraction of the quaternionic geometry relations yields another version of the central charge modified $\lambda$-world HK constraints, with $\tilde{z}_{A}^{0}$ included into the set of analytic space coordinates. The final results, however, are the same as in the version worked out in [2]. Without entering into details, we only mention the basic 
redefinitions one should make to ensure an unambigous passing to the limit $R \rightarrow 0$ in the $\lambda$-world relations deduced in the previous Section

$$
H^{+4}=R \tilde{H}^{+4}, \quad \mathcal{L}_{\mu}^{+}=R \tilde{\mathcal{L}}_{\mu}^{+}, \quad \mathcal{L}^{+4}=R \tilde{\mathcal{L}}^{+4} .
$$

Finally, let us quote some basic objects of quaternionic geometry in the HK limit $R \rightarrow 0$

$$
\begin{aligned}
H^{+3 \nu} & \Rightarrow-\frac{1}{2} \tilde{T}^{\nu \mu}\left(\partial_{\mu}^{-} \tilde{\mathcal{L}}^{+4}-\partial^{++} \tilde{\mathcal{L}}_{\mu}^{+}\right) \\
\omega_{\alpha \beta}^{++} & \Rightarrow e_{(\alpha}^{\mu} \Delta^{++} e_{\mu \beta)}+e_{(\alpha}{ }^{\nu} \partial_{\nu}{ }^{-} H^{+3 \mu} e_{\mu \beta)} \\
g_{(\lambda)}^{\mu+\nu-} & \Rightarrow-\tilde{T}^{\mu \rho}\left(\partial H^{-1}\right)_{\rho}{ }^{\nu} \\
g_{(\lambda)}^{\mu-\nu-} & \Rightarrow 2 \tilde{T}^{\rho \lambda}\left(\partial H^{-1}\right)_{\lambda}{ }^{\sigma}\left(\partial H^{-1}\right)_{\rho}{ }^{(\mu} \partial_{\sigma}^{+} H^{-3 \nu)}, \quad \text { etc. }
\end{aligned}
$$

Thus, as it should be, in the limit when the $S p(1)$ curvature vanishes, one self-consistently recovers the unconstrained harmonic space formulation of HK manifolds.

\section{Geometry of $N=2$ matter in $N=2$ supergravity background}

Here we demonstrate that the equations of motion following from the general harmonic superspace off-shell action of $N=2$ matter coupled to $N=2$ supergravity given in [10] have a simple interpretation in terms of quaternionic geometry in the unconstrained harmonic space formulation presented above. Surprisingly, the components

$$
\omega=u_{i}^{-} q^{+i}, \quad N^{++}=\frac{u_{i}^{+} q^{+i}}{u_{j}^{-} q^{+j}}
$$

of the supergravity hypermultiplet compensator $q^{+i}$ acquire a clear geometric meaning as the coordinates $z_{A}^{0}, z_{A}^{++}$of the analytic space $\left\{z_{A}^{0}, z_{A}^{++}, w^{ \pm i}, x^{\mu+}\right\}$ of the quaternionic geometry, while $x^{\mu+}$ (like in the HK case) are identified with the $N=2$ matter hypermultiplet superfileds.

Let us start with the $N=2$ matter - supergravity action [9] written in terms of compensators $\left.\omega, N^{++} 110.1\right)$ 18]

$$
\begin{gathered}
S_{S G+\text { matter }}^{N=2}=-\frac{1}{2 \kappa^{2}} \int d \zeta^{(-4)} d u \omega^{2}\left\{\mathcal{H}^{+4}-\mathcal{D}_{u}^{++} N^{++}-\left(N^{++}\right)^{2}\right. \\
\left.-\frac{\kappa^{2}}{\xi^{2}}\left[\tilde{\mathcal{L}}^{+4}(Q, w)+\tilde{\mathcal{L}}^{+\mu}(Q, w) \mathcal{D}_{w}^{++} Q_{\mu}^{+}\right]\right\} \\
w^{-i}=u^{-i}, \quad w^{+i}=u^{+i}-N^{++} u^{-i}, \quad \mathcal{D}_{w}^{++}=\mathcal{D}_{u}^{++}+N^{++} \mathcal{D}^{0} .
\end{gathered}
$$

In (10.2), integration goes over the analytic subspace of harmonic $N=2$ superspace, $Q^{+}$are $N=2$ matter hypermultiplet analytic superfields, $\mathcal{H}^{+4}$ is the analytic harmonic vielbein of conformal $N=2$ supergravity, $\mathcal{D}_{u}^{++}$is the supergravity-covariantized harmonic 
derivative with respect to $u^{ \pm i}$ in the analytic basis of $N=2$ harmonic superspace and $\kappa, \xi$ are, respectively, Newton and sigma-model coupling constants (for details see ref. 3, 9, 10, 18)

The action (10.2) is invariant [10] under two kinds of gauge transformations with analytic parameters: diffeomorphisms (the parameters $\left.\lambda^{+\mu}(Q, w)\right)$ and the quaternionic analog of the HK and Kähler transformations (the parameters $\left.\Lambda^{++}(Q, w)\right)$ :

$$
\begin{gathered}
\delta Q^{+\mu}=\lambda^{+\mu}(Q, w) \\
\delta N^{++}=-\frac{\kappa^{2}}{\xi^{2}} \Lambda^{++}, \quad \delta \omega=\frac{1}{2} \frac{\kappa^{2}}{\xi^{2}}\left[-\partial_{w}^{--} \Lambda^{++}-\tilde{\mathcal{L}}_{\mu}^{+} \partial_{w}^{--} \lambda^{+\mu}\right] \omega \\
\delta \tilde{\mathcal{L}}_{\mu}^{+}=-\hat{\partial}_{\mu}^{-} \Lambda^{++}-\tilde{\mathcal{L}}_{\rho}^{+} \hat{\partial}_{\mu}^{-} \lambda^{+\rho}, \quad \delta \mathcal{D}_{w}^{++}=-\frac{\kappa^{2}}{\xi^{2}} \Lambda^{++} \mathcal{D}^{0} \\
\delta \tilde{\mathcal{L}}^{+4}=\partial_{w}^{++} \Lambda^{++}+\tilde{\mathcal{L}}_{\mu}^{+} \partial_{w}^{++} \lambda^{+\mu} \\
+\frac{\kappa^{2}}{\xi^{2}}\left[\partial_{w}^{--} \Lambda^{++} \tilde{\mathcal{L}}^{+4}+\partial_{w}^{--} \lambda^{+\mu} \tilde{\mathcal{L}}_{\mu}^{+} \tilde{\mathcal{L}}^{+4}-\Lambda^{++} \tilde{\mathcal{L}}_{\mu}^{+} Q^{+\mu}\right] \\
\hat{\partial}_{\mu}^{-} \equiv \partial_{\mu}^{-}-\frac{\kappa^{2}}{\xi^{2}} \tilde{\mathcal{L}}_{\mu}^{+} \partial_{w}^{--} .
\end{gathered}
$$

Identifying

$$
\begin{aligned}
Q^{+\mu} & \equiv x_{A}^{+\mu}, \frac{\kappa^{2}}{\xi^{2}} \Lambda^{++} \equiv-\lambda^{++}, \quad \frac{\kappa^{2}}{\xi^{2}} \tilde{\mathcal{L}}_{\mu}^{+} \equiv-\mathcal{L}_{\mu}^{+} \\
\frac{\kappa^{2}}{\xi^{2}} \tilde{\mathcal{L}}^{+4} & \equiv \mathcal{L}^{+4}, \omega \equiv z_{A}^{0}, \quad N^{++} \equiv z_{A}^{++}, \frac{\kappa^{2}}{\xi^{2}} \equiv-R
\end{aligned}
$$

we observe the one-to-one correspondence between $(10.4)-(10.7)$ and the quaternionic geometry transformation laws in the gauge $\tilde{e}=\tilde{B}$ (7.17) (eqs. (5.6), (6.35), (7.9)). We see that this correspondence requires $R<0$ i.e. in the case at hand, while considering homogeneous quaternionic manifolds, one necessarily deals with their noncompact versions (see Sect. 11). Recalling (2.10), one recovers the familiar relation [8, 14] between the coupling constant $\frac{\kappa^{2}}{\xi^{2}}$ and the scalar curvature of the quaternionic manifold of $N=2$ matter

$$
R_{\alpha i \beta j}{ }^{\alpha i \beta j}=-8 n(n+2) \frac{\kappa^{2}}{\xi^{2}} .
$$

Thus the basic entities of the most general $N=2$ matter action in the supergravity background, $\tilde{\mathcal{L}}_{\mu}^{+}$and $\tilde{\mathcal{L}}^{+4}$, are recognized as the unconstrained quaternionic geometry potentials, the matter superfields $Q^{+}$being the coordinates of the analytic subspace of harmonic extension of the target quaternionic manifold. This makes evident the one-toone correspondence between off-shell locally $N=2$ supersymmetric sigma models and quaternionic manifolds revealed at the component on-shell level in 8 .

\footnotetext{
${ }^{1}$ The full matter-supergravity action also includes the purely supergravity part, which, however, is irrelevant here to our purposes.
} 
Let us now, as we promised, give the geometric interpretation to the equations of motion following from (10.2).

Varying (10.2) with respect to $\omega, N^{++}$and $Q^{+\mu}$ yields, respectively,

$$
\begin{aligned}
\delta \omega: & \mathcal{D}_{u}^{++} N^{++}+\left(N^{++}\right)^{2}-\mathcal{H}^{+4}+\left(\mathcal{L}^{+4}+\mathcal{L}_{\mu}^{+} \mathcal{D}_{w}^{++} Q^{+\mu}\right)=0 \\
\delta N^{++}: & N^{++}-\mathcal{D}_{u}^{++} \ln \omega-\frac{1}{2} \Gamma^{++}+\frac{1}{2} \mathcal{L}_{\mu}^{+} Q^{+\mu} \\
& -\frac{1}{2}\left(\partial_{w}^{--} \mathcal{L}^{+4}+\partial_{w}^{--} \mathcal{L}_{\mu}^{+} \mathcal{D}_{w}^{++} Q^{+\mu}\right)=0 \\
\delta Q^{+\mu}: & \partial_{\mu}^{-} \mathcal{L}^{+4}+\left(\partial_{\mu}^{-} \mathcal{L}_{\rho}^{+}-\partial_{\rho}^{-} \mathcal{L}_{\mu}^{+}\right) \mathcal{D}_{w}^{++} Q^{+\rho}-\partial_{w}^{++} \mathcal{L}_{\mu}^{+} \\
& +2 \mathcal{L}_{\mu}^{+}\left(N^{++}-\mathcal{D}_{u}^{++} \ln \omega-\frac{1}{2} \Gamma^{++}\right) \\
& -\left[\mathcal{H}^{+4}-\mathcal{D}_{u}^{++} N^{++}-\left(N^{++}\right)^{2}\right] \partial_{w}^{--} \mathcal{L}_{\mu}^{+}=0 .
\end{aligned}
$$

Here $\Gamma^{++}$is the $L(1)$ connection of conformal $N=2$ supergravity (it is expressed in a proper way through the analytic $N=2$ supergravity prepotentials, its precise form is of no interest for us) and we passed to renormalized objects (10.8). Substituting (10.10), (10.11) into eq. (10.12), one recasts the latter into the form

$$
\left(\hat{\partial}_{\mu}^{-} \mathcal{L}_{\rho}^{+}-\hat{\partial}_{\rho}^{-} \mathcal{L}_{\mu}^{+}\right) \mathcal{D}_{w}^{++} Q^{+\rho}=-\hat{\partial}_{\mu}^{-} \mathcal{L}^{+4}+\mathcal{L}_{\mu}^{+}\left(\mathcal{L}_{\rho}^{+} Q^{+\rho}\right)+\partial_{w}^{++} \mathcal{L}_{\mu}^{+}+\mathcal{L}^{+4} \partial_{w}^{--} \mathcal{L}_{\mu}^{+}
$$

that is precisely the relation (7.10) upon the identification

$$
\mathcal{D}_{w}^{++} Q^{+\rho}=\mathcal{D}_{u}^{++} Q^{+\rho}+N^{++} Q^{+\rho}=H^{+3 \rho} .
$$

Recall that the analogous interpretation, but in the framework of the harmonic space formulation of HK geometry, can be given to the $Q^{+}$equations following from the general off-shell action of sigma model with rigid $N=2$ supersymmetry [2].

It remains to clarify the geometric meaning of the relations (10.10), (10.11) and (10.14) which involve the objects $N^{++}, \omega$ having no prototypes in the harmonic superspace action of rigid $N=2$ matter. While the identification (10.14) has a direct analog in the rigid case (with $\mathrm{N}^{++}$put equal zero), the remaining two relations are essentially new. Remarkably, for all of them one can find a natural place within the quaternionic geometry formulation presented in this paper.

We note first that the counterpart of the derivative $D^{++}(5.9)$ is just $\mathcal{D}_{u}^{++}$: it is invariant under the $\lambda^{+\mu}$ and $\lambda^{++}$invariances and transforms only under gauge group of conformal $N=2 \mathrm{SG}$. In other words, it can be regarded as the $N=2 \mathrm{SG}$ gauge covariantization of $D^{++}(5.9)$. Then $\mathcal{D}_{w}^{++}$corresponds to $\mathcal{D}_{\lambda}^{++}$defined in (5.10). Let us now consider the action of $D^{++}$(5.9) on $z_{A}^{++}$:

$$
D^{++} z_{A}^{++}=-\left[H^{+4}+\left(z_{A}^{++}\right)^{2}\right] .
$$

Comparing it with (10.10) and taking into account the relation (7.8)

$$
H^{+4}=\mathcal{L}^{+4}+\mathcal{L}_{\mu}^{+} \mathcal{D}_{w}^{++} Q^{+\mu}=\mathcal{L}^{+4}+\mathcal{L}_{\mu}^{+} H^{+3 \mu}
$$

and the identifications (10.8), one concludes that (10.10) is nothing else as the $N=2 \mathrm{SG}$ covariantization of the quaternionic geometry relation (10.15). One simply adds to $H^{+4}$ 
the $N=2 \mathrm{SG}$ vielbein $\mathcal{H}^{+4}$, thus ensuring covariance both under the quaternionic and $N=2$ SG gauge transformations.

Analogously, eq.(10.11) can be rewritten as

$$
\begin{aligned}
\mathcal{D}_{u}^{++} \omega= & -\frac{1}{2}\left[\partial_{w}^{--} \mathcal{L}^{+4}+\partial_{w}^{--}\left(\mathcal{L}_{\mu}^{+} \mathcal{D}_{w}^{++} Q^{+\mu}\right)-\mathcal{L}_{\mu}^{+} \partial_{w}^{--}\left(\mathcal{D}_{w}^{++} Q^{+\mu}\right)-\mathcal{L}_{\mu}^{+} Q^{+\mu}\right] \omega \\
& +\left(N^{++}-\frac{1}{2} \Gamma^{++}\right) \omega
\end{aligned}
$$

Comparing it with eq.(7.25), we can rewrite it as

$$
\mathcal{D}_{u}^{++} \omega=\left(N^{++}+\phi^{++}-\frac{1}{2} \Gamma^{++}\right) \omega
$$

that is a direct $N=2 \mathrm{SG}$ covariantization of the relation

$$
D^{++} z_{A}^{0}=\left(z_{A}^{++}+\phi^{++}\right) z_{A}^{0}
$$

following from the explicit form of $D^{++}(5.9)$.

Finally, the relation (10.14) can also be geometrically interpreted as the result of action of $D^{++}$(5.9) on $x^{+\mu}$ :

$$
D^{++} x^{+\mu}=H^{+3 \mu}-z_{A}^{++} x^{+\mu} .
$$

Thus we have proved complete one-to-one correspondence between the unconstrained formulation of quaternionic geometry given in the previous Sections and the equations of motion of general $N=2$ sigma model coupled to $N=2 \mathrm{SG}$.

We stress that the $S p(1)$ coordinates $z_{A}^{++}=N^{++}$and $z_{A}^{0}=\omega$ play the important role both in the harmonic superspace geometry of $N=2 \mathrm{SG}$ and in the harmonic space geometry of $N=2$ matter superfields. They are common for both geometries and so establish a link between them.

\section{Potentials for symmetric quaternionic spaces}

In this Section we quote the explicit form of the potentials $\mathcal{L}^{+4}$ for all types of connected symmetric quaternionic spaces classified in [11] 2.

There are precisely one compact and one noncompact quaternionic cosets for each simple complex Lie group. Here we specialize to the noncompact versions of them which one encounters in $N=2$ supergravity.

These cosets are 11, 12]

$$
\begin{array}{ccccc}
\frac{S U(n, 2)}{U(n) \times S p(1)} & \frac{S O(n, 4)}{S O(n) \times S U(2) \times S p(1)} & \frac{S p(n, 1)}{S p(n) \times S p(1)} & \frac{G_{2(+2)}}{S U(2) \times S p(1)} \\
\frac{F_{4(+4)}}{S p(3) \times S p(1)} & \frac{E_{6(+2)}}{S U(6) \times S p(1)} & \frac{E_{7(-5)}}{S O(12) \times S p(1)} & \frac{E_{8(-24)}}{E_{7} \times S p(1)}
\end{array}
$$

They have the form $G / H \times S p(1)$ with $H \in S p(n)$. The number in brackets for the exceptional groups refers to the chosen real form of the group. It equals to the difference

\footnotetext{
${ }^{2} \mathrm{~A}$ more general class of homogeneous nonsymmetric quaternionic spaces has been given in [12].
} 
between the numbers of noncompact and compact generators for this real form. The compact case corresponds to the compact real form of the numerator.

As explained above the coset $S p(n, 1) / S p(n) \times S p(1)$ is a "flat model" for the quaternionic geometry. The corresponding $\mathcal{L}^{+4}$ vanishes. The potentials for the cosets

$$
\frac{S U(n, 2)}{U(n) \times S p(1)} \quad \frac{S O(n, 4)}{S O(n) \times S U(2) \times S p(1)} \quad \frac{G_{2(+2)}}{S U(2) \times S p(1)}
$$

were given in 10. Here we recall these potentials and complete the list. We give only the final expressions for $\mathcal{L}^{+4}$. The general procedure of finding them based on the analogy with the Hamiltonian mechanics [20] has been recently worked out in [21].

The coset parameters $Q^{i \Sigma}$ for manifolds (11.1) have the quantum numbers of the group generators belonging to the coset: they always carry the index $i(i=1,2)$ of the fundamental two-dimensional representation of the group $S p(1)$ from the denominator $H \times S p(1)$ and the index $\Sigma(\Sigma=1,2, \ldots 2 n)$ which refers to the group $H$. The potential $\mathcal{L}^{+4}$ depends only on the '+'-projection $Q^{+\Sigma}$ of $Q^{i \Sigma}$. In the sequel we will refer just to $Q^{+\Sigma}$ as the coset parameters. Clearly, as $H$ defines a linear symmetry on $Q_{\Sigma}^{+}$and $\mathcal{L}^{+4}$ is $H$ invariant, one needs to know the assignment of the adjoint representation of the group in the nominator, $G$, with respect to the subgroup $H$.

We briefly sketch how $\mathcal{L}^{+4}$ can be found within the geometric set-up employed in previous Sections. Computations are based on several simplifying observations.

Firstly, since in the above cosets linearly realized $S p(1)$ acts, the relevant potentials $\mathcal{L}^{+4}$ do not depend explicitly on the harmonics $w_{i}^{ \pm}$. Then the condition

$$
\mathcal{D}^{0} \mathcal{L}^{+4}=4 \mathcal{L}^{+4}
$$

tells us that these potentials must be quartic in $Q_{\Sigma}^{+}$10.

Secondly, the group $G$ defines isometries of these cosets, so there exist Killing potentials $K_{A}^{++}$, the number of which is equal to $\operatorname{dim} G$ and which are solutions of eq. (7.56). For the case at hand this equation simplifies to

$$
\partial^{++} K^{++}+\left(\partial^{--} K^{++}\right) \mathcal{L}^{+4}+\frac{1}{2} \hat{\partial}^{-\mu} K^{++} \hat{\partial}_{\mu}^{-} \mathcal{L}^{+4}=0
$$

(for simplicity, we put $R=-1$ in accordance with the fact that we consider here only noncompact manifolds). The $S p(1)$ Killing potential $K_{i j}^{++}$has the universal form for all spaces $(11.1)$

$$
K_{i j}^{++}=w_{i}^{+} w_{j}^{+}+w_{i}^{-} w_{j}^{-} \mathcal{L}^{+4},
$$

while inspection of eq. (11.3) for the symmetries belonging to the coset and the subgroup $H$ leads to the important equation

$$
Q_{\Sigma}^{+} \mathcal{L}^{+4}-\frac{1}{4} \Omega^{\Lambda \Gamma} \frac{\partial^{2} \mathcal{L}^{+4}}{\partial Q^{+\Sigma} \partial Q^{+\Lambda}} \frac{\partial \mathcal{L}^{+4}}{\partial Q^{+\Gamma}}=0
$$

Here $\Omega^{\Sigma \Gamma}$ is the invariant antisymmetric $S p(n)$ tensor.

It turns out that the equation (11.4), together with the requirement of $H$-invariance of $\mathcal{L}^{+4}$ and the property that $\mathcal{L}^{+4}$ is a quartic polynomial in $Q_{\Sigma}^{+}$, determine $\mathcal{L}^{+4}$ uniquely: for 
each case a $H$-invariant polynomial of degree 4 is unique up to a numerical normalization factor, which is fixed by eq. (11.4).

1. $S U(n, 2) / U(n) \times S p(1)$. The coset parameters are $\left(Q_{a}^{+}, \bar{Q}^{+a}\right)$, where $a$ is the index of the fundamental representation of $U(n)$. The pseudoreal structure is given by

$$
* Q_{a}^{+}=\bar{Q}^{+a}, \quad * \bar{Q}^{+a}=-Q_{a}^{+} .
$$

The potential $\mathcal{L}^{+4}$ is

$$
\mathcal{L}^{+4}=-\left(Q^{+} \bar{Q}^{+}\right)^{2}
$$

2. $S O(n, 4) / S O(n) \times S U(2) \times S p(1)$. The coset parameters are $Q_{a \alpha}^{+}$where $a$ is the vector index of $S O(n)$ and $\alpha$ is the spinor index of $S U(2)$. The pseudoreal structure is given by:

$$
* Q_{a \alpha}^{+}=Q_{a}^{+\alpha}
$$

The potential is

$$
\mathcal{L}^{+4}=\frac{1}{2}\left(Q_{a \alpha}^{+} Q_{b}^{+\alpha}\right)\left(Q_{\gamma}^{+a} Q^{+b \gamma}\right)
$$

3. $G_{2(+2)} / S U(2) \times S p(1)$. Now the coset parameters are $Q_{\alpha \beta \gamma}^{+}$, where greek indices refer to the spinor representation of $S U(2)$. They are totally antisymmetric in $\alpha, \beta$, and $\gamma$. Pseudoreality:

$$
* Q_{\alpha \beta \gamma}^{+}=Q^{+\alpha \beta \gamma}
$$

The potential is

$$
\mathcal{L}^{+4}=-\frac{1}{2} Q^{+\alpha \beta \gamma} Q_{\alpha \beta \rho}^{+} Q^{+\sigma \tau \rho} Q_{\sigma \tau \gamma}^{+}
$$

4. $F_{4(+4)} / S p(3) \times S p(1)$. The coset parameters transform as components of a traceless antisymmetric tensor of rank 3 for $S p(3)$. In other words,

$$
Q^{+\alpha \beta \gamma}=Q^{+[\alpha \beta \gamma]} \text { with } \Omega_{\alpha \beta} Q^{+\alpha \beta \gamma}=0
$$

where greek indices refer to the fundamental three-dimensional representation of $S p(3)$ and $\Omega_{\alpha \beta}$ is the defining symplectic form. Pseudoreality:

$$
* Q_{\alpha \beta \gamma}^{+}=Q^{+\alpha \beta \gamma} \text {. }
$$

Potential:

$$
\mathcal{L}^{+4}=-\frac{3}{2} Q^{+\alpha \beta \gamma} Q_{\alpha \beta \rho}^{+} Q^{+\sigma \tau \rho} Q_{\sigma \tau \gamma}^{+}
$$

5. $E_{6(+2)} / S U(6) \times S p(1)$. In this case the coset parameters $Q^{+\alpha \beta \gamma}$ are described by the rank 3 antisymmetric tensor of $S U(6)$. The pseudoreality conditions and the potential look exactly as in the previous case:

$$
\begin{gathered}
* Q_{\alpha \beta \gamma}^{+}=Q^{+\alpha \beta \gamma} \\
\mathcal{L}^{+4}=-\frac{3}{2} Q^{+\alpha \beta \gamma} Q_{\alpha \beta \rho}^{+} Q^{+\sigma \tau \rho} Q_{\sigma \tau \gamma}^{+} .
\end{gathered}
$$


6. $E_{7(-5)} / S O(12) \times S p(1)$. The coset parameters $Q_{\alpha}^{+}$transform as components of a chiral spinor of the group $S O(12)$. Pseudoreality:

$$
* Q_{\alpha}^{+}=Q^{+\alpha}
$$

The potential is

$$
\mathcal{L}^{+4}=\frac{9}{432} \Sigma_{m n}^{\alpha \beta} \Sigma_{m n}^{\gamma \rho} Q_{\alpha}^{+} Q_{\beta}^{+} Q_{\gamma}^{+} Q_{\rho}^{+}
$$

Here

$$
\Sigma_{m n}^{\alpha \beta}=\frac{1}{2}\left(\Sigma_{m \dot{\beta}}^{\alpha} \Sigma_{n}^{\dot{\beta} \beta}-\Sigma_{n \dot{\beta}}^{\alpha} \Sigma_{m}^{\dot{\beta} \beta}\right)
$$

and $\Sigma_{m \alpha \dot{\beta}}$ are $\sigma$-matrices in 12 dimensions. Indices are raised and lowered with the charge conjugation matrix $C^{\alpha \beta}$ which is antisymmetric in 12 dimensions.

7. $E_{8(-24)} / E_{7} \times S p(1)$. The coset parameters $Q_{\Sigma}^{+}$are transformed according to the representation $\underline{56}$ of $E_{7}$. It is convenient to decompose it with respect to the maximal subgroup $S U(8) \subset E_{7}$. It breaks into antisymmetric tensors $Q_{i j}^{+}$and $Q^{+i j}$, where $i$ is the index of the fundamental representation of $S U(8)$. Pseudoreality:

$$
* Q_{i j}^{+}=\bar{Q}^{+i j}, \quad * \bar{Q}^{+i j}=-Q_{i j}^{+}
$$

The potential takes the form

$$
\mathcal{L}^{+4}=-4 Q_{i k}^{+} Q^{+i l} Q^{+}{ }_{n l} Q^{+n k}+\left(Q_{i j}^{+} Q^{+i j}\right)^{2}-\frac{1}{4 !}\left(\Phi^{+4}+\bar{\Phi}^{+4}\right)
$$

where

$$
\Phi^{+4}=\epsilon^{i_{1} j_{1} i_{2} j_{2} i_{3} j_{3} i_{4} j_{4}} Q_{i_{1} j_{1}}^{+} Q_{i_{2} j_{2}}^{+} Q_{i_{3} j_{3}}^{+} Q_{i_{4} j_{4}}^{+} .
$$

The general formula for all these $\mathcal{L}^{+4} \mathrm{~s}$ in terms of the structure constants of $G$ was given in [21]. It reads as follows

$$
\mathcal{L}^{+4}=\frac{1}{3}(n+2) g^{a b} K_{a}^{++} K_{b}^{++} .
$$

Here $g^{a b}$ is the restriction of the inverse Killing-Cartan metric of $G$ to $H$ and $K_{a}^{++}$is the Killing potential associated with $H$. The specifity of the cases presented above is encoded (through the structure constants of $G$ ) in these objects.

An interesting (though straightforward) problem is to explicitly compute the quaternionic $\tau$ world metrics for the above $\mathcal{L}^{+4}$ 's like this has been done for the simplest case $S p(n+1) / S p(n) \times S p(1)$ in Sect. 8 .

\section{Conclusions}

In the series of papers including [1], 2] and the present article we have given a complete description of self-dual Yang-Mills theory, hyper-Kähler and quaternionic geometries in the universal language of harmonic analyticity. The latter underlies both these purely bosonic theories and their supersymmetric counterparts: $N=2$ Yang-Mills theory and 
$N=2$ supergravity [3, 9, 10], thus establishing a deep affinity between these two classes of theories, so different at first sight. Moreover, as we have seen in [2] and in this paper, the uniform harmonic (super)space description of these theories allows to understand in a most geometric fashion why $N=2$ supersymmetry requires the target manifolds of $N=2$ supersymmetric sigma models to be hyper-Kähler or quaternionic. It seems that this universality of the harmonic (super)space approach is its main merit. This approach is most efficient just while exploring the relationships between the target space geometries and space-time extended supersymmetries. As an example of its such recent application we mention the analysis of the target space geometry of $2 D N=(4,0)$ sigma models in [19]. In contrast to the cases considered here and in [2], this geometry is non-Riemannian, the torsion essentially enters into game. However, the harmonic space techniques proved to be adequate for this case too.

One of intriguing problems for the future study is to find out possible implications of $N=3$ harmonic analyticity [22] in the purely bosonic gauge theories. The geometry underlied by this type of analyticity (if exists) is expected to be intimately related to $N=3$ supersymmetric theories: $N=3$ Yang-Mills theory [22 and supergravity.

\section{Acknowledgements}

We sincerely thank V.I. Ogievetsky for interest in the work and stimulating and useful discussions. A.G. acknowledges support by the U.S. National Science Foundation, grant PHY-90096198. E.I. thanks ENSLAPP in Lyon for hospitality.

\section{References}

[1] A. Galperin, E. Ivanov, V. Ogievetsky and E. Sokatchev, Ann. Phys. (N.Y.) 185 (1988) 1.

[2] A. Galperin, E. Ivanov, V. Ogievetsky and E. Sokatchev, Ann. Phys. (N.Y.) 185 (1988) 22.

[3] A. Galperin, E. Ivanov, S. Kalitzin, V. Ogievetsky and E. Sokatchev, Class. Quantum Grav. 1 (1984) 469.

[4] L. Alvarez-Gaume and D.Z. Freedman, Commun. Math. Phys. 80 (1981) 443.

[5] A. Galperin, E. Ivanov, V. Ogievetsky and E. Sokatchev, Commun. Math. Phys. 103 (1986) 515.

[6] A. Galperin, E. Ivanov and V. Ogievetsky, Nucl. Phys. B 282 (1987) 74.

[7] B. Zumino, Phys. Lett. B 87 (1979) 203.

[8] J. Bagger and E. Witten, Nucl. Phys. B 222 (1983) 1.

[9] A. Galperin, E. Ivanov, V. Ogievetsky and E. Sokatchev, Class. Quantum Grav. 4 (1987) 1255. 
[10] J.A. Bagger, A.S. Galperin, E.A. Ivanov and V.I. Ogievetsky, Nucl. Phys. B 303 (1988) 522.

[11] J. Wolf, J. Math. Mech. 14 (1965) 1033.

[12] D.A. Alekseevskii, Math. USSR Izvestija 9 (1975) 297.

[13] S. Salamon, Invent. Math. 67 (1982) 143.

[14] J. Bagger, in Supersymmetry, ed. K. Dietz, R. Flume, G. Gehlen and V. Rittenberg (Plenum, New York) 1985.

[15] A.L. Besse, Einstein Manifolds, Springer, Berlin, 1987, vol. 1.

[16] R. Penrose, Gen. Rel. Grav. 7 (1976) 31.

[17] R.S. Ward, Phys. Lett. 61 B (1977) 81; M.F. Atiyah and R.S. Ward, Commun. Math. Phys. 55 (1977) 117.

[18] E. Ivanov, Phys. Lett. B 205 (1988) 499.

[19] F. Delduc, S. Kalitzin and E. Sokatchev, Class. Quantum Grav. 7 (1990) 1567.

[20] A.Galperin and V. Ogievetsky, Class. Quantum Grav. 8 (1991) 1757.

[21] A. Galperin and O. Ogievetsky, Harmonic potentials for quaternionic symmetric $\sigma$ models, preprint JHU-TIPAC-920022, MPI-Ph/92-84.

[22] A. Galperin, E. Ivanov, S. Kalitzin, V. Ogievetsky and E. Sokatchev, Phys. Lett. B 151 (1985) 215; Class. Quantum Grav. 2 (1985) 155. 